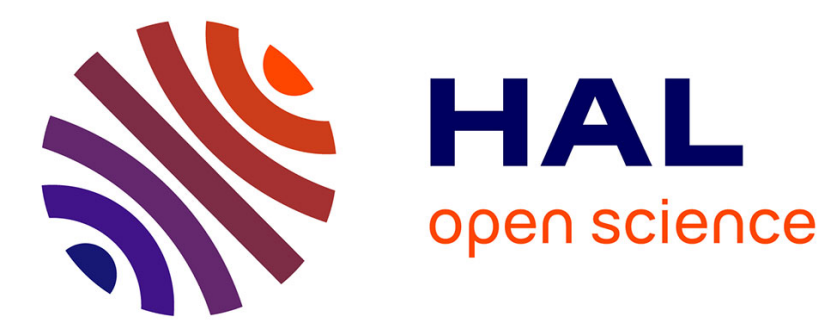

\title{
Phenomenological coefficients in a concentrated alloy for the dumbbell mechanism
}

Vincent Barbe, Maylise Nastar

\section{To cite this version:}

Vincent Barbe, Maylise Nastar. Phenomenological coefficients in a concentrated alloy for the dumbbell mechanism. Philosophical Magazine, 2006, 86 (23), pp.3503-3535. 10.1080/14786430600654420 . hal00513683

\section{HAL Id: hal-00513683 \\ https://hal.science/hal-00513683}

Submitted on 1 Sep 2010

HAL is a multi-disciplinary open access archive for the deposit and dissemination of scientific research documents, whether they are published or not. The documents may come from teaching and research institutions in France or abroad, or from public or private research centers.
L'archive ouverte pluridisciplinaire HAL, est destinée au dépôt et à la diffusion de documents scientifiques de niveau recherche, publiés ou non, émanant des établissements d'enseignement et de recherche français ou étrangers, des laboratoires publics ou privés. 


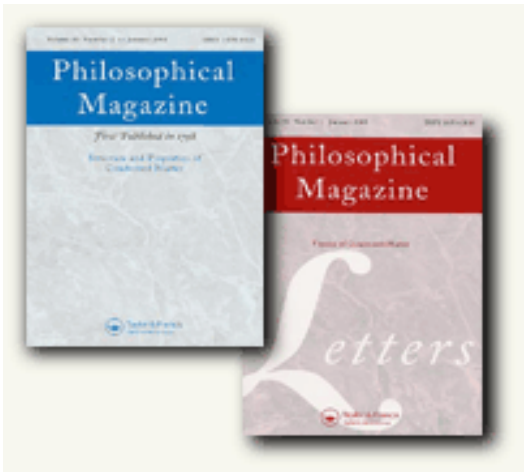

\section{Phenomenological coefficients in a concentrated alloy for the dumbbell mechanism}

\begin{tabular}{|r|l|}
\hline Journal: & Philosophical Magazine \& Philosophical Magazine Letters \\
\hline Manuscript ID: & TPHM-05-Dec-0560.R1 \\
\hline Journal Selection: & Philosophical Magazine \\
\hline Date Submitted by the & 21-Feb-2006 \\
\hline Keywords: & $\begin{array}{l}\text { transport, alloys, correlated systems, diffusion, defects in solids, } \\
\text { multiscale modelling }\end{array}$ \\
\hline Keywords (user supplied): & mean field, dumbbell \\
\hline & $\begin{array}{l}\text { Barbe, Vincent; CEA Saclay, DMN/SRMP } \\
\text { Nastar, Maylise; CEA Saclay, DMN/SRMP }\end{array}$ \\
\hline $\begin{array}{l}\text { Note: The following files were submitted by the author for peer review, but cannot be converted } \\
\text { to PDF. You must view these files (e.g. movies) online. }\end{array}$ \\
\hline $\begin{array}{l}\text { maintext.tex } \\
\text { appendix.tex } \\
\text { main.tex } \\
\text { barbib.bib } \\
\text { figures.tex }\end{array}$ \\
\hline
\end{tabular}

\section{$\checkmark$ ScholaroNE" \\ Manuscript Central}




\title{
Phenomenological coefficients in a concentrated alloy for the dumbbell mechanism
}

\author{
V. Barbe, M. Nastar \\ Service de Recherches en Métallurgie Physique, CEA/Saclay, \\ 91191 Gif-sur-Yvette Cedex, France.
}

\begin{abstract}
We present an adaptation of the self-consistent mean field (SCMF) theory to calculate the transport coefficients in a concentrated alloy for diffusion by the dumbbell mechanism. In this theory, kinetic correlations are accounted for through a set of effective interactions within a non-equilibrium distribution function of the system. Transport coefficients are calculated for the FCC and BCC multicomponent concentrated alloys for simple sets of jump frequencies, including different stabilities of the different defects. A first approximation leads to an analytical expression of the Onsager coefficients in a binary alloy, and a second approximation provides with a more accurate prediction. The results of the SCMF theory are compared with existing models and available Monte Carlo simulations, and an interpretation of the set of effective interactions in terms of a competition between jump frequencies is proposed.
\end{abstract}

Keywords : Diffusion ; Dumbbell ; Concentrated alloy ; Correlations ; Mean Field.

Short title : Transport coefficients for the dumbbell mechanism

*Author for correspondence. Email : vincent.barbe@cea.fr. http://mc.manuscriptcentral.com/pm-pml 


\section{Introduction}

When an alloy is irradiated with particles, atomic transport can occur through both types of defects which are created, vacancies and interstitials. The calculation of the transport coefficients associated to both mechanisms was therefore intensively studied in the past decades, mainly to predict the variation of atomic and defect fluxes with the concentration and the basic jump frequencies. In the case of the vacancy mechanism, relatively complete models are now available, including effects as a percolation threshold $[1,2,3]$ or dependence of the transport coefficient with the thermodynamic interactions between atoms $[4,5,6,7]$. However, due to its greater complexity, an efficient general model is still lacking for the dumbbell mechanism.

Bocquet $[8,9,10]$ was the first one to propose a calculation of the tranport coefficients for the dumbbell mechanism in concentrated alloys. He used an approach based on the description of macrojumps, i.e. a series of consecutive jumps of the defect in which one particular atom does not leave the defect : based on this description, he calculated the tracer diffusion coefficients in a face-centered cubic (FCC) concentrated alloy and followed a procedure close to the one of Manning [1] for the vacancy mechanism to deduce the Onsager coefficients $[8,9]$. This method, also called effective field approximation, could predict a percolation behaviour for high jump frequency ratios. This approximation happened to be not applicable in BCC alloys [10]. Nevertheless, Monte Carlo simulations were developed in both systems to provide with a reference value of the transport coefficients $[9,10,11]$.

Some years later, Chaturvedi and Allnatt $[12,13]$ derived the calculation of the $L_{i j}$ coefficients within the framework of equilibrium fluctuations, based on the successful earlier work of Moleko, Allnatt and Allnatt [2] in the vacancy case. Their results in terms of tracer [12] and collective [13] correlation factors appeared to be more or less accurate than those of Bocquet compared to the Monte Carlo simulations as a function of the chosen set of jump frequencies for a global jump frequency ratio of 1:10, although Bocquet's model involved a less complex formalism. One has also to notice that http://mc.manuscriptcentral.com/pm-pml 
the transport coefficients obtained by this method do not fulfill the Onsager law, i.e. the Onsager matrix is not symmetrical, which makes the choice of the coefficient $L_{A B}$ problematic.

Recently, Sharma et al. [14] adapted the same formalism to the BCC concentrated alloy, but the geometrical complexity of the jump mechanism in this particular structure prevented them to complete the calculation of the transport coefficients. However, they could derive useful relations between the correlation coefficients, in good agreement with Monte Carlo simulations.

The model of jump frequencies used in those studies is a simplified set of frequencies proposed by Bocquet [9], which allows dumbbells of different compositions to present different binding energies. Interactions between a dumbbell and a substitutional atom as well as between two substitutional atoms were neglected : one must notice that the formalism of Chaturvedi and Allnatt does not allow such interactions, whereas Bocquet's model could integrate them at the cost of a great additional complexity. Sets of jump frequencies including interactions between defects and substitutionnal atoms were introduced up to now only in the case of dilute alloys for the dumbbell mechanism : among others, Barbu [15] and Allnatt et al. [16] treated this case with the pair association method [17] in a FCC dilute alloy. However, later works on dilute alloys [18, 19, 20] neglected the interactions between dumbbells and solute atoms.

More generally, it must be noted that the assumptions in terms of geometry of the defect and of the jump mechanism can still be discussed : in this study we apply the widely accepted models proposed in a review by Robrock [21] and confirmed by recent ab initio calculations in the BCC structure $[22,23]$, but such calculations in FCC alloys are still missing, and all results were obtained up to now in pure elements or dilute alloys. Geometrical details for each structure will be addressed in their corresponding section.

In this paper we address the calculation of the transport coefficients in a general concentrated alloy using a self-consistent mean field (SCMF) theory $[4,24,5,3]$ developped in the past years for 
the case of the vacancy mechanism. It is based on an atomic model of the jump mechanism, and introduces a non-equilibrium density function through a set of 'effective' interactions which are calculated thanks to particular kinetic equations derived from a Master Equation in presence of chemical potential gradients, under steady state conditions [4]. Recently, it was successfully extended to treat a percolation threshold in concentrated alloys [3] as well as a complete thermodynamic description of a dilute alloy including atomic interactions [5]. Adapting the SCMF to the dumbbell mechanism does not involve much change in the formalism, apart from a greater geometrical complexity.

In a first part, we present the general formalism of the SCMF in the dumbbell case (section 2). Detailed calculations are presented to enlighten the differences from the vacancy mechanism treated with the same formalism. In the two following sections, we will show its application to noninteracting * concentrated alloys : the cases of FCC and BCC structures, intensively discussed in the litterature, appeared very similar in terms of treatment and results, both are therefore presented in the same article. The last section will be devoted to the comparison between the SCMF results and the existing models, as well as general remarks on the SCMF formalism. Results of the SCMF theory in the case of a dilute alloy will be presented soon in a following paper : the authors found more consistent to present the structure of the method in the general case of a concentrated alloy where the symmetries are more likely to be emphasized and take advantage of simple analytic expressions of the transport coefficients under some simplifying assumptions.

\section{The SCMF theory}

The main steps of the calculation of the transport coefficients using the SCMF theory in the case of a dumbbell mechanism are the same as for the vacancy mechanism, each one requiring only light

\footnotetext{
*Throughout this paper, we call non-interacting a system without interactions involving the substitutional atoms. As we will see, a particular set of jump frequencies can take into account different stabilities of the dumbbells of different compositions, which is equivalent to speak of thermodynamic interactions between both atoms inside the dumbbell. Nevertheless, non-interacting alloys in this study are characterised by the absence of short-range or long-range order.
} 
modifications. The main assumption of the model is to introduce a non-equilibrium formalism in terms of configuration probability, which depends on a driving force, here a gradient of chemical potential. The dumbbell mechanism which gives rise to diffusion is described by a Master Equation involving the non-equilibrium distribution function. In a second part, we introduce an atomic model of the jump frequencies. Eventually, we present the final system of kinetic equations which leads to the calculation of transport coefficients. As the spirit of the calculation is exactly the same as for the vacancy mechanism, we will emphasize in the following paragraphs the characteristic aspects induced by the dumbbell mechanism and will not detail all calculations, which are fully described in earlier papers $[4,5,3]$.

\subsection{Non-equilibrium distribution}

We consider a system of atoms and dumbbells distributed on the $N_{s}$ sites of a rigid lattice. A configuration $\mathbf{n}$ of the system is described by the occupation numbers $\left\{n_{1}^{A}, n_{1}^{B}, \ldots n_{1}^{A B_{\alpha}}, \ldots n_{2}^{A}, n_{2}^{B} \ldots\right\}$ : $n_{i}^{A}$ is equal to 1 when the site $i$ is occupied by a substitutional atom $A$ and 0 if else. Here a defect $A B_{\alpha}$ is defined as two atoms $A$ and $B$ occupying the same lattice site, $\alpha$ standing for the orientation of the dumbell as well as its direction if $A$ is different from $B$. Generalized occupation numbers are introduced for convenience. On the one hand, $N_{i}^{A}$ is the total number of atoms of the species A occupying the lattice site $i$. This generalized occupation number can take the values 0,1 or 2 and is defined as :

$$
N_{i}^{A}=n_{i}^{A}+2 \times \sum_{\alpha} n_{i}^{A A_{\alpha}}+\sum_{\alpha, B \neq A} n_{i}^{A B_{\alpha}}
$$

On the other hand, $N_{i}^{I}$ is an occupation number of the site $i$ by whatever a dumbbell :

$$
N_{i}^{I}=\sum_{\alpha, A, B} n_{i}^{A B_{\alpha}}
$$

Throughout this paper, any sum over chemical species will not include the dumbbells, unless explicitely mentioned.

http://mc.manuscriptcentral.com/pm-pml 
In a first approximation, in contrary to the SCMF theory with vacancy mechanism, we shall consider a non-interacting system, as even such simple systems are currently not satisfactorily treated by available theories. As a consequence, the equilibrium probability of one configuration $\mathbf{n}$ reduces to :

$$
\widehat{P}_{0}=\exp \left[\beta\left(\Omega+\sum_{A, i} \mu_{A} N_{i}^{A}+\mu_{I} N_{i}^{I}\right)\right] .
$$

The quantity $\beta=1 / k_{B} T$ is the reciprocal temperature weighted by the Boltzmann constant, $\Omega$ is the grand canonical potential which guarantees the relationship $\sum_{\mathbf{n}} \widehat{P}_{0}(\mathbf{n})=1$ and $\mu_{A}$ is the chemical potential of the species $A$. Note that the chemical potentials of all species including dumbbells are not independent because of the property of the occupation numbers :

$$
\sum_{A} N_{i}^{A}-N_{i}^{I}=1
$$

Out of equilibrium, we define the new probability of a configuration $\mathbf{n}$ by the product of an equilibrium and a non-equilibrium part :

$$
\widehat{P}(\mathbf{n}, t)=\widehat{P}_{0}(\mathbf{n}) \widehat{P}_{1}(\mathbf{n}, t)
$$

where the non-equilibrium contribution has the characteristic form :

$$
\widehat{P}_{1}(\mathbf{n}, t)=\exp \left[\beta\left(\delta \Omega(t)+\sum_{A, i} \delta \mu_{i}^{A}(t) N_{i}^{A}+\sum_{i} \delta \mu_{i}^{I}(t) N_{i}^{I}-\hat{h}(t)\right)\right]
$$

Following Vaks [25], in addition to the corrections to the chemical potentials and to the grand canonical potential due to non-equilibrium, we introduce an unknown time-dependent function which states a new set of correlations between the occupation numbers. This function $\hat{h}(t)$ is chosen to have the form of an Hamiltonian as a function of the occupation numbers and will be called effective Hamiltonian. In the case of the dumbbell mechanism, it should include interactions between both species inside a dumbbell as well as interactions between the dumbbell and the substitutional atoms.

$$
\hat{h}(t)=\sum_{\text {http: } I / \text { mc.manuscripptecentral.com/pm-pml }} v_{i}^{I}(t) n_{i}^{I}+\frac{1}{2 !} \sum_{i j} v_{i j}^{A I}(t) n_{i}^{A} n_{j}^{I}+\ldots
$$


where the sum over $I$ means a sum over all types of interstitial defects (composition and orientation). The unknown time-dependent functions $v_{i j \ldots}^{\alpha \beta \ldots}$ are called for convenience effective interactions although their physical meaning is in no way comparable to thermodynamic interactions. As it was the case with the vacancy mechanism, effective interactions between more than two atoms or dumbbells may also be introduced, which give rise to a more accurate description of long paths of the defect.

\subsection{Atomic diffusion model for the dumbbell mechanism}

The unknown effective interactions will be related to the transport coefficients of the system by modelling its kinetic behaviour. Following Vaks [25], we use the Master Equation :

$$
\frac{d \widehat{P}(\mathbf{n}, t)}{d t}=\sum_{\tilde{\mathbf{n}}}[\widehat{W}(\tilde{\mathbf{n}} \rightarrow \mathbf{n}) \widehat{P}(\tilde{\mathbf{n}}, t)-\widehat{W}(\mathbf{n} \rightarrow \tilde{\mathbf{n}}) \widehat{P}(\mathbf{n}, t)]
$$

where $\widehat{W}(\mathbf{n} \rightarrow \tilde{\mathbf{n}})$ is the probability of the transition from the configuration $\mathbf{n}$ to the configuration $\tilde{\mathbf{n}}$ per time unit. In the case of the dumbbell mechanism, a transition from one configuration to another one can be composed of either a rotation of a dumbbell $A B_{\alpha}$ on its site (becoming $A B_{\beta}$ ), or of a single jump of the dumbbell. It is widely assumed that, in the latter case, one atom of the dumbbell migrates to a neighbouring 'target site' and forms a new dumbbell with the species which was already on this site ('target atom'). The remaining atom of the initial dumbbell is then left in a substitutional position.

$$
A B_{\alpha}+C \rightarrow A+B C_{\beta}
$$

Note that the use of the character $\beta$ in subscript or superscript always refers to an orientation/direction, whereas the same character in normal police stands for the inverse temperature multiplied by the Boltzmann constant. The probability of such a jump exchange per time unit is :

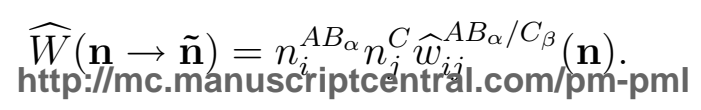


Several simplified sets of jump frequencies have been proposed by different authors. The most widely used is the one by Bocquet $[26,9]$, which assumes that the jump frequency depends only on the three species $A, B$ and $C$ and can be written $w_{A B / C}$. However, even in a binary alloy, this model can lead to eight different frequencies : simplified models with only two jump frequencies have been used by Bocquet $[9,10]$ and Sharma et al. [20]. The former assumed that the frequency is controlled by the change (or not) of the composition of the dumbbell during the jump, introducing different stabilities of the different dumbbells ; the latter proposed a more 'vacancy-like' model where the jump frequency depends only upon the migrating species. More sophiscated models were used in the case of dilute alloys (see for example Barbu [15] or Allnatt et al. [16]) where the formation and dissociation of pairs containing one dumbbell and one solute atom were considered. Throughout this paper, we assume a set of frequencies of the type $w_{A B / C}^{\alpha \beta}$ as introduced by Bocquet and present the numerical results in the more convenient two-frequency model. On-site rotation is also considered with a frequency $w_{A B}^{R}$ depending only on the composition of the rotating dumbbell : again, more complex models can be necessary if one considers a binding energy between dumbbells and neighbouring atom, which will not be the case here.

An overview of the possible orientations $\alpha$ given the cristallographic structure was proposed by Robrock [21] and was since supported by ab initio calculations $[22,23]$. Hence, relations between the initial and final orientations $\alpha$ and $\beta$ in Equation (9) also depend on the cristallographic structure, as well as the definition of target sites for one initial orientation. As long as the structure is not mentioned, we define a factor $\gamma_{i j}^{\alpha \beta}$ which is equal to 1 if the jump described by equation (9) is allowed, and equal to 0 if this jump is forbidden for geometrical reasons. Eventually we can write the basic jump frequency as :

$$
\widehat{w}_{i j}^{A B_{\alpha} / C_{\beta}}(\mathbf{n})=\gamma_{i j}^{\alpha \beta} w_{A B / C}^{\alpha \beta}
$$

In the RHS, the 'hat' sign on $w$ has been removed because this frequency does not depend on the http://mc.manuscriptcentral.com/pm-pml 
configuration of the system excepted for the nature of the three involved atoms $A, B$ and $C$. We note that, defining $\bar{\beta}$ as the direction opposite to $\beta$, the latter factor obeys the relation :

$$
\gamma_{i j}^{\alpha \beta}=\gamma_{j i}^{\bar{\beta} \bar{\alpha}}
$$

and the RHS is to be applied to the reverse jump :

$$
C B_{\bar{\beta}}+A \rightarrow C+B A_{\bar{\alpha}}
$$

where we have used the equality $A B_{\alpha}=B A_{\bar{\alpha}}$. A similar factor $\gamma_{R}^{\alpha \beta}$ is introduced to separate among the rotations between orientations $\alpha$ and $\beta$ those which are geometrically allowed $\left(\gamma_{R}^{\alpha \beta}=1\right)$ and those which are forbidden $\left(\gamma_{R}^{\alpha \beta}=0\right)$. This factor depends on the cristallographic structure and will be made explicit in the application of the SCMF model to FCC and BCC structures. Throughout this article, applications will focus on the $\langle 110\rangle$ dumbbell in the BCC structure and the $\langle 100\rangle$ dumbbell in the FCC one. Target sites as well as useful notations for some important configurations are summarized in figures 1 and 2 for the FCC and BCC structures respectively.

\subsection{System of kinetic equations}

For the calculation of the transport coefficients, we consider now the particular case of a system out of equilibrium, with the following conditions :

- The system is near an homogeneous equilibrium state, so that the non-equilibrium quantities are close to zero.

- Thanks to suitable boundary conditions, the driving force is an homogeneous chemical potential gradient.

- The system is assumed to be in steady state.

In these conditions, we solve the Master Equation in order to calculate the unknown effective interactions.

http://mc.manuscriptcentral.com/pm-pml 
Instead of searching $\widehat{P}$ as a solution of the Master Equation, we define it by its moments. Unlike the vacancy mechanism, we will focus the calculation on the moments involving the defect, which are of the type $\left\langle n_{i}^{A B_{\alpha}}\right\rangle$ for a one-point moment and $\left\langle n_{i}^{A B_{\alpha}} n_{j}^{C}\right\rangle$ for a two-point moment. The kinetic equation of the type of Equation (8) is applied to those moments. It comes for example:

$$
\begin{aligned}
\frac{d\left\langle n_{i}^{A B_{\alpha}}\right\rangle}{d t}= & \sum_{s \neq i, \beta, \sigma}\left\langle n_{s}^{\sigma A_{\beta}} n_{i}^{B} \gamma_{s i}^{\beta \alpha} w_{\sigma A / B}^{\beta \alpha}+n_{s}^{C B_{\beta}} n_{i}^{A} \gamma_{s i}^{\beta \bar{\alpha}} w_{\sigma B / A}^{\beta \bar{\alpha}}\right. \\
& \left.-n_{i}^{A B_{\alpha}} n_{s}^{\sigma} \gamma_{i s}^{\bar{\alpha} \beta} w_{B A / \sigma}^{\bar{\alpha} \beta}-n_{i}^{A B_{\alpha}} n_{s}^{\sigma} \gamma_{i s}^{\alpha \beta} w_{A B / \sigma}^{\alpha \beta}\right\rangle \\
& +\sum_{\beta}\left\langle\left(\gamma_{R}^{\beta \alpha} n_{i}^{A B_{\beta}}-\gamma_{R}^{\alpha \beta} n_{i}^{A B_{\alpha}}\right) w_{R}^{A B}\right\rangle .
\end{aligned}
$$

Here the first two terms refer to the migration of an existing dumbbell towards the site $i$ initially occupied by a species $B$ (respectively $A$ ) ; the following two terms refer to the migration of the dumbell from the site $i$ to a target neighbour site $s$, leaving on site $i$ the $B$ atom (respectively $A$ ). The last two terms refer to the appearance or disappearance of the dumbbell $A B_{\alpha}$ by means of a rotation. The kinetic equation for the two-point moment $\left\langle n_{i}^{A B_{\alpha}} n_{j}^{C}\right\rangle$ is exactly of the same type, but attention should be paid to the possible jump of the dumbbell from site $i$ to site $j$. It comes :

$$
\begin{aligned}
\frac{d\left\langle n_{i}^{A B_{\alpha}} n_{j}^{C}\right\rangle}{d t}= & \left\langle\sum _ { \beta , \sigma , s \neq i , j } \left( n_{j}^{C} n_{s}^{\sigma A_{\beta}} n_{i}^{B} \gamma_{s i}^{\beta \alpha} w_{\sigma A / B}^{\beta \alpha}+n_{j}^{C} n_{s}^{\sigma B_{\beta}} n_{i}^{A} \gamma_{s i}^{\beta \bar{\alpha}} w_{\sigma B / A}^{\beta \bar{\alpha}}\right.\right. \\
& \left.-n_{j}^{C} n_{i}^{A B_{\alpha}} n_{s}^{\sigma} \gamma_{i s}^{\bar{\alpha} \beta} w_{B A / \sigma}^{\bar{\alpha} \beta}-n_{j}^{C} n_{i}^{A B_{\alpha}} n_{s}^{\sigma} \gamma_{i s}^{\alpha \beta} w_{A B / \sigma}^{\alpha \beta}\right) \\
& +\sum_{\beta}\left(n_{j}^{C A_{\beta}} n_{i}^{B} \gamma_{j i}^{\beta \alpha} w_{C A / B}^{\beta \alpha}+n_{j}^{C B_{\beta}} n_{i}^{A} \gamma_{j i}^{\beta \bar{\alpha}} w_{C B / A}^{\beta \bar{\alpha}}\right. \\
& \left.-n_{i}^{A B_{\alpha}} n_{j}^{C} \gamma_{i j}^{\bar{\alpha} \beta} w_{B A / C}^{\bar{\alpha} \beta}-n_{i}^{A B_{\alpha}} n_{j}^{C} \gamma_{i j}^{\alpha \beta} w_{A B / C}^{\alpha \beta}\right) \\
& \left.+\sum_{\beta} n_{j}^{C}\left(\gamma_{R}^{\beta \alpha} n_{i}^{A B_{\beta}}-\gamma_{R}^{\alpha \beta} n_{i}^{A B_{\alpha}}\right) w_{R}^{A B}\right\rangle
\end{aligned}
$$

where terms involving the presence of two dumbbells have been neglected.

The next step is the linearization with respect to the terms $\beta\left(\delta \mu_{i}^{A}-\delta \mu_{s}^{A}\right)$ and $\beta \hat{h}$. This calculation is detailed in Appendix A : it is greatly simplified by the use of the detailed balance, which is satisfied 
at equilibrium and states for the migration and the rotation of the dumbbell respectively :

$$
\begin{aligned}
\left\langle n_{s}^{C A_{\beta}} n_{i}^{B} \gamma_{s i}^{\beta \alpha} w_{C A / B}^{\beta \alpha}\right\rangle^{(0)} & =\left\langle n_{i}^{A B_{\alpha}} n_{s}^{C} \gamma_{i s}^{\bar{\alpha} \beta} w_{B A / C}^{\bar{\alpha} \beta}\right\rangle^{(0)} \\
\left\langle\gamma_{R}^{\beta \alpha} n_{i}^{A B}{ }^{A B} w_{R}^{A B}\right\rangle^{(0)} & =\left\langle\gamma_{R}^{\alpha \beta} n_{i}^{A B}{ }^{A B} w_{R}^{A B}\right\rangle^{(0)}
\end{aligned}
$$

where \langle\rangle$^{(0)}$ means an equilibrium average over the equilibrium distribution fonction $P_{0}$.

Eventually, each moment set to zero on one or many sites leads to a linear relation between the effective interactions and the gradients of chemical potentials of the different species.

\subsection{Transport coefficients and correlation effects}

The link with the transport coefficients is based on the time derivative of the one-point moment of the generalized occupation number $\left\langle N_{i}^{A}\right\rangle$. Given its definition, any variation of $N_{i}^{A}$ can occur only if an atom jumps to or from site $i$ :

$$
\begin{aligned}
\frac{d\left\langle N_{i}^{A}\right\rangle}{d t}= & \sum_{s \neq i, \alpha, \beta, \sigma, \sigma^{\prime}}\left\langle n_{s}^{\sigma^{\prime} A_{\beta}} n_{i}^{\sigma} \gamma_{s i}^{\beta \alpha} w_{\sigma^{\prime} A / \sigma}^{\beta \alpha}\right. \\
& \left.-n_{i}^{\sigma A_{\bar{\alpha}}} n_{s}^{\sigma^{\prime}} \gamma_{i s}^{\bar{\alpha} \bar{\beta}} w_{\sigma A / \sigma^{\prime}}^{\bar{\alpha} \bar{\beta}}\right\rangle .
\end{aligned}
$$

This moment is identified as a local concentration $\left\langle N_{i}^{A}\right\rangle \equiv c_{i}^{A}$. One recognizes the equation of matter conservation :

$$
\frac{d c_{i}^{A}}{d t}=-\sum_{s \neq i} J_{i \rightarrow s}^{A}
$$

where the flux of atoms $A$ from site $i$ to site $j$ is :

$$
\begin{aligned}
J_{i \rightarrow s}^{A} & =\sum_{\alpha \beta, \sigma, \sigma^{\prime}}\left\langle n_{i}^{\sigma A_{\alpha}} n_{s}^{\sigma^{\prime}} \gamma_{i s}^{\alpha \beta} w_{\sigma A / \sigma^{\prime}}^{\alpha \beta}-n_{s}^{\sigma^{\prime} A_{\bar{\beta}}} n_{i}^{\sigma} \gamma_{s i}^{\bar{\beta} \bar{\alpha}} w_{\sigma A / \sigma^{\prime}}^{\bar{\beta} \bar{\alpha}}\right\rangle \\
& =-\beta \sum_{\alpha, \beta, \sigma, \sigma^{\prime}} \gamma_{i s}^{\alpha \beta} w_{\sigma A / \sigma^{\prime}}^{\alpha \beta}\left\langle n_{i}^{\sigma A_{\alpha}} n_{s}^{\sigma^{\prime}}\left(\mu_{s}^{A}-\mu_{i}^{A}+\hat{h}_{i}^{\sigma A_{\alpha}}-\hat{h}_{s}^{A \sigma_{\beta}^{\prime}}\right)\right\rangle
\end{aligned}
$$

where $\hat{h}_{i}^{\sigma A_{\alpha}}$ is the partial derivative of $\hat{h}$ with respect to the occupation number $n_{i}^{\sigma A_{\alpha}}$. The procedure to obtain the second line is described in detail in Appendix A, and will be used in the same manner in all kinetic moments. 
It will now be convenient to write the flux directly in terms of the effective interactions :

$$
J_{i \rightarrow s}^{A}=-\beta l_{A}^{(0)}\left(\mu_{s}^{A}-\mu_{i}^{A}\right)-\beta \mathbf{l}_{\mathbf{A}} \times \mathbf{V}
$$

where $\mathbf{V}$ is the column vector of the effective interactions and $\mathbf{l}_{\mathbf{A}}$ a line vector of the same dimension.

We can then identify the transport coefficients of the system regarding the species $A$ as the coefficients of proportionnality relating the flux of atoms $A$ and the gradients of chemical potentials of each species $\sigma$, i.e. the projection of the RHS of equation (21) along the vector $\left(\mu_{s}^{\sigma}-\mu_{i}^{\sigma}\right)$. We can separate the uncorrelated part of the coefficient $L_{A A}^{\dagger}$ as :

$$
\frac{1}{\beta} L_{A A}^{(0)}=\left\langle\sum_{\alpha, \beta, \sigma, \sigma^{\prime}} n_{i}^{\sigma A_{\alpha}} n_{j}^{\sigma^{\prime}} \gamma_{i s}^{\alpha \beta} w_{\sigma A / \sigma^{\prime}}^{\alpha \beta}\right\rangle^{(0)}
$$

In opposition to the vacancy mechanism, it is possible to restrict the effective Hamiltonian to interactions within one site only, i.e. the effective interactions of the type $v_{i}^{I}$. In this case, Equation (20) is greatly simplified and one can express the correlated transport coefficient as :

$$
\sum_{\alpha, \beta, \sigma, \sigma^{\prime}} \gamma_{i s}^{\alpha \beta} w_{\sigma A / \sigma^{\prime}}^{\alpha \beta}\left\langle n_{i}^{\sigma A_{\alpha}} n_{s}^{\sigma^{\prime}}\left(\hat{h}_{i}^{\sigma A_{\alpha}}-\hat{h}_{s}^{A \sigma_{\beta}^{\prime}}\right)\right\rangle=\frac{1}{\beta} \sum_{B}\left(\delta_{A B} L_{A A}^{(0)}-L_{A B}\right)\left(\mu_{s}^{B}-\mu_{i}^{B}\right)
$$

Throughout the article, we found more convenient to express the transport coefficients in terms of correlation coefficients $f_{A B}^{(A)}$ which follow the usual definition :

$$
f_{A B}^{(A)}=\frac{L_{A B}}{L_{A A}^{(0)}}
$$

where the superscipt ${ }^{(A)}$ is omitted if $A=B$.

\subsection{Effective interactions in the case of one-dimensional diffusion}

After solving the kinetic equations, one obtains the expression of the effective interactions as a linear combination of the gradients of chemical potentials of the atomic species (see for example $[4,5,3]$ for

\footnotetext{
${ }^{\dagger}$ The link with the macroscopic transport phenomenology for a cubic symmetry states: $\tilde{J}_{A}=\sum_{\sigma}\left(a^{2} / V_{a t}\right) L_{A \sigma} \nabla \mu_{\sigma}$, where $\tilde{J}_{A}$ is expressed in atoms per time and area units, $a$ is the lattice parameter and $V_{a t}$ the atomic volume.

http://mc.manuscriptcentral.com/pm-pml
} 
such expressions in the vacancy case). It is then straightforward to deduce that in these conditions :

$$
v_{i j}^{A B}=-v_{j i}^{A B}
$$

where $A$ and $B$ are subsitutional atoms. Namely, inverting sites $i$ and $j$ is equivalent to invert the sign of the gradients of chemical potentials. Concerning the dumbbells, attention must be paid to apply a complete symmetric inversion, involving the exchange of sites and the orientation of the dumbbell. Let us note $\bar{I}$ the 'inversed' dumbbell (defined by $\overline{A B_{\alpha}}=A B_{\bar{\alpha}}=B A_{\alpha}$ ) :

$$
\begin{aligned}
v_{i}^{\bar{I}} & =-v_{i}^{I} \\
v_{i j}^{A \bar{I}} & =-v_{i j}^{I A}
\end{aligned}
$$

The consequence is a considerable reduction of the number of independent effective interactions. On the one hand, interactions of the type $v_{i}^{A B_{\alpha}}$ only need to be taken into account if $A$ is different from $B$ and if the orientation $\alpha$ is not perpendicular to the main direction of diffusion which is chosen to be the direction $\langle 100\rangle$ (also called $X$ axis). The contribution of the dumbbell $A B$ will be noted $v_{A B}$ if the projection of $\alpha$ along the direction $X$ is positive (the dumbbell can be noted $A B^{+}$), and $v_{B A}=-v_{A B}$ if the projection is negative. If the projection is zero (dumbbell $A B^{0}$ ), so is the corresponding effective interaction.

Concerning the interactions involving one defect $A B_{\alpha}$ on site $i$ and one substitutionnal atom $C$ on site $j$, we will limit ourselves in this study to nearest neighbour (nn) interactions. For symmetry reasons, it is sufficient to take into account configurations where the projection of the vector $i j$ along the $X$ direction is positive or equal to zero. To this point, it will be necessary to differentiate if site $j$ is a possible target site of the dumbbell or not. Four possibilities arise which are summed up in figures 1 and 2 for the case of FCC and BCC structures respectively. We use the subscripts $\|$ if the jump $A B \rightarrow C$ is allowed ( $C$ is on a compression site) and $\perp$ if the jump is forbidden ( $C$ on a dilatation site), and the superscript + (respectively 0) stands for an orientation of the dumbbell $A B$ positive 
along the $X$ direction (respectively perpendicular to the $X$ direction) : effective interactions one has to deal with are of the type $v_{A B}, v_{A B_{\|}^{+} C}, v_{A B_{\perp}^{+} C}, v_{A B_{\|}^{0} C}$ and $v_{A B_{\perp}^{0} C}$. Examples of the antisymmetry properties in the BCC structure are given in figure 3. Eventually, if the projection of the dumbbell and of the vector $i j$ on the $X$ axis are both 0 , the associated effective interaction is also 0 . In a system containing $n$ atomic species, the number of effective interactions (and of kinetic equations to solve) is $n(n-1) / 2+4 n^{3}$ : the first term comes from the effective interactions inside a dumbbell $A B^{+}$and the second term stands for the four independent positions of a complex $A B_{\alpha} C$. For each cristallographic structure we will derive the calculation using this entire set of kinetic equations and address eventually the possible simplifications and their consequences in terms of transport coefficients.

\section{[ Insert Figure 1 about here. ] \\ [ Insert Figure 2 about here. ] \\ [ Insert Figure 3 about here. ]}

\section{$3 \quad$ FCC concentrated alloy}

\subsection{Jump mechanism}

In a FCC alloy the most stable configuration of a dumbbell is the $\langle 100\rangle$ direction. The jump mechanism as described among others by Johnson [27] is a jump towards a nn atom including a rotation of $90^{\circ}$ of the orientation of the dumbbell. On-site rotation of the dumbbell with an angle of $90^{\circ}$ is also allowed.

The twelve nn atoms of an $A B$ dumbbell must then be separated into four target sites for the atom $A$, four target sites for the atom $B$ and four sites which cannot be reached in one jump. Figure 1 summarizes the different configurations to be taken into account.

http://mc.manuscriptcentral.com/pm-pml 
Eventually, we define equilibrium concentrations of the different dumbbells as :

$$
c_{A B}=\left\langle n_{i}^{A B_{\alpha}}\right\rangle^{(0)}
$$

The quantity $c_{A A}$ is then one third of the total concentration of dumbbells of type $A A$, and $c_{A B}$ is one sixth of the total concentration of mixed dumbbells, with the relation :

$$
\sum_{A, B} c_{A B}=\frac{c_{I}}{3}
$$

due to the three possible orientations of the dumbbell. The way to calculate $c_{A B}$ is detailed in Appendix B.

To simplify the notations, we introduce an effective field $h_{A B}$ as the total effective interactions involved by a single dumbbell $A B$ in an average environment :

$$
h_{A B}=v_{A B}+4 \sum_{\sigma}\left(v_{A B_{\|}^{+} \sigma}-v_{B A_{\|}^{+} \sigma}+v_{A B_{\perp}^{+} \sigma}\right) c_{\sigma}
$$

and express the moments in terms of $h_{A B}$ instead of $v_{A B}$. We will also use the compact notation :

$$
v_{A B_{\|}^{+} \bar{C}}=\sum_{\sigma} v_{A B_{\|}^{+} \sigma} c_{\sigma}-v_{A B_{\|}^{+} C}
$$

The way to obtain such combinations of effective interactions is detailed in Appendix C.

Eventually, we introduce weighted jump frequencies $W_{A B / C}$, which are proportional to the probability of a jump $A B \rightarrow C$ in the system :

$$
W_{A B / C} \equiv c_{A B} c_{C} w_{A B / C}
$$

as well as mean jump frequencies of a certain dumbbell :

$$
W_{A B / X} \equiv c_{A B} \sum_{\sigma} c_{\sigma} w_{A B / \sigma}
$$

and finally the average jump frequency of one species :

$$
W_{A} \equiv \sum_{\sigma, \sigma^{\prime}} c_{\sigma^{\prime} A} c_{\sigma} w_{\sigma^{\prime} A / \sigma} .
$$

The corresponding frequency for rotation is $W_{A B}^{R} \equiv c_{A B} w_{A B}^{R}$. http://mc.manuscriptcentral.cóm/pm-pml 


\subsection{Kinetic equations}

The kinetic equations of the one-point and two-point moments are much more complex than the ones obtained for the vacancy mechanism, because of the additional geometry and of the increased number of atoms involved in one jump. We will detail the calculation of the one-point moment to show the origin of the different terms and give the expression of three more characteristic two-point moments, the other being of the very same structure is given in Appendix D.

We first express the flux of atoms $A$ by adapting Equation (20) to the FCC structure :

$$
\begin{aligned}
J_{i \rightarrow j+}^{A}= & -2 W_{A}\left(\mu_{j+}^{A}-\mu_{i}^{A}\right) \\
& -\sum_{\sigma, \sigma^{\prime}} c_{\sigma A} c_{\sigma^{\prime}}\left(h_{\sigma A}-v_{\sigma A_{\|}^{+} \overline{\sigma^{\prime}}}-v_{\sigma A_{\|}^{0} \overline{\sigma^{\prime}}}\right. \\
& \left.-h_{A \sigma^{\prime}}+v_{\sigma^{\prime} A_{\|}^{+} \bar{\sigma}}+v_{\sigma^{\prime} A_{\|}^{0} \bar{\sigma}}\right) w_{\sigma A / \sigma^{\prime}} \\
= & -2 W_{A}\left(\mu_{j+}^{A}-\mu_{i}^{A}\right) \\
& -2 \sum_{\sigma, \sigma^{\prime}}\left(h_{\sigma A}-v_{\left.\sigma A_{\|}^{+} \overline{\sigma^{\prime}}-v_{\sigma A_{\|}^{0} \overline{\sigma^{\prime}}}\right) W_{\sigma A / \sigma^{\prime}} .}\right.
\end{aligned}
$$

To obtain the latter expression, we use the equality $W_{\sigma A / \sigma^{\prime}}=W_{\sigma^{\prime} A / \sigma}$ deduced from the detailed balance and invert the order of $\sigma$ and $\sigma^{\prime}$ in the double sum.

We now express Equation (14) in terms of the simplified effective interactions in a FCC lattice. Considering the dumbbell $A B$ with a positive orientation on site $i, j+$ is a target site for the jump $A B / C$ and $j-$ a target site for the jump $B A / C$ (see figure 1 ). The four possible positions of $j+$ or $j-$ are all equivalent due to the one-dimensional symmetry.

$$
\begin{aligned}
\frac{d\left\langle n_{i}^{A B^{+}}\right\rangle}{d t}= & \left\langle n_{i}^{B} \sum_{\sigma} 4 n_{j-}^{\sigma A^{0}} w_{\sigma A / B}\right. \\
& +n_{i}^{A} \sum_{\sigma} 4 n_{j+}^{B \sigma^{0}} w_{\sigma B / A} \\
& -n_{i}^{A B^{+}} \sum_{\sigma}\left[4 n_{j-}^{\sigma} w_{B A / \sigma}+4 n_{j+}^{\sigma} w_{A B / \sigma}\right] \\
& \left.+\left(4 n_{i}^{A B^{0}}-4 n_{i}^{A B^{+}}\right) w_{R}\right\rangle .
\end{aligned}
$$

http://mc.manuscriptcentral.com/pm-pml 
Applying the same procedure as in Appendix A, we derive it in terms of non-equilibrium quantities :

$$
\begin{aligned}
\frac{d\left\langle n_{i}^{A B^{+}}\right\rangle}{d t}= & 4 W_{A B / X}\left(\mu_{j+}^{B}-\mu_{i}^{B}\right)-4 W_{B A / X}\left(\mu_{i}^{A}-\mu_{j-}^{A}\right) \\
& +4\left(W_{B A / X}+W_{A B / X}+W_{A B}^{R}\right) h_{A B} \\
& -4 \sum_{\sigma}\left[W_{A B / \sigma}\left(v_{A B_{\|}^{+} \bar{\sigma}}+v_{\sigma B_{\|}^{0} \bar{A}}\right)\right. \\
& \left.-W_{B A / \sigma}\left(v_{B A_{\|}^{+} \bar{\sigma}}+v_{\sigma A_{\|}^{0} \bar{B}}\right)\right]
\end{aligned}
$$

We give the expression of three more 2-point moments over the four possible ones to illustrate particular points of the calculation. The last one is formally very close to the latters and is to be found in Appendix D.

$$
\begin{aligned}
\frac{d\left\langle n_{i}^{A B_{\|}^{+}} n_{j}^{C}\right\rangle}{d t}= & -4 c_{C} W_{B A / X}\left(\mu_{i}^{A}-\mu_{j-}^{A}\right)+\left(3 c_{C} W_{A B / X}+W_{A B / C}\right)\left(\mu_{j+}^{B}-\mu_{i}^{B}\right) \\
& -4 \sum_{\sigma} W_{B A / \sigma} c_{C}\left(-v_{\sigma A_{\|}^{0} \bar{B}}-v_{B A_{\|}^{+} \bar{\sigma}}-h_{A B}+v_{A B_{\|}^{+} \bar{C}}\right) \\
& -3 \sum_{\sigma} W_{A B / \sigma} c_{C}\left(v_{\sigma B_{\|}^{0} \bar{A}}+v_{A B_{\|}^{+} \bar{\sigma}}-h_{A B}+v_{A B_{\|}^{+} \bar{C}}\right) \\
& -W_{A B / C}\left(v_{C B_{\|}^{+} \bar{A}}-h_{A B}+v_{A B_{\|}^{+} \bar{C}}\right) \\
& +c_{C} W_{A B}^{R}\left(v_{A B_{\|}^{0} \bar{C}}+v_{B A_{\|}^{0} \bar{C}}+2 v_{A B_{\perp}^{0} \bar{C}}+4 h_{A B}-4 v_{A B_{\|}^{+} \bar{C}}\right) W_{A B}^{R} .
\end{aligned}
$$

Here the effective interactions $h$ and $v$ have been grouped as a function of the mechanism of appearance/disappearance of the complex $A B_{\|}^{+} C$. The second line refers to a jump of atom $A$ toward or from the dumbbell (four possible sites), the third line to a jump of atom $B$ involving a site other than $j$ (three possible sites), the fourth line to a jump of $B$ between sites $i$ and $j$, and the fifth line to an on-site rotation. Furthermore, we note for convenience $n_{i j}^{A B_{\|}^{+} C}$ the product $n_{i}^{A B} n_{j}^{C}$ where atoms $A, B$ and $C$ are in a particular configuration which gives rise to the effective interaction $v_{A B_{\|}^{+} C}:$ an example of such a configuration is given in figure 1(e). 
In the first and second lines of the RHS, one can perceive the principal effect contained in the 2-point moment, which is the competition for a dumbbell $A B$ between the jump toward a given species (here $C$ ) and the jump into an average medium. This is more visible if we express the ratio of the generalized frequencies $W$ as :

$$
\begin{aligned}
\frac{W_{A B / C}}{c_{C}\left(4 W_{A B / X}+3 W_{B A / X}\right)} & =\frac{c_{A B} c_{C} w_{A B / C}}{c_{C} \sum_{\sigma} c_{A B} c_{\sigma}\left(4 w_{A B / \sigma}+3 W_{B A / \sigma}\right)} \\
& =\frac{w_{A B / C}}{\sum_{\sigma} c_{\sigma}\left(w_{A B / \sigma}+W_{B A / \sigma}\right)}
\end{aligned}
$$

The last 2-moment equations concern a substitutionnal atom in a nn non-target site. It is convenient to use instead of the two-point moment the quantity $K_{i j}^{A B_{\perp}^{+} C} \equiv\left\langle n_{i j}^{A B_{\perp}^{+} C}-n_{i}^{A B^{+}} c_{C}\right\rangle$.

$$
\begin{aligned}
& \frac{d K_{i j}^{A B_{\perp}^{+} C}}{d t}=-4 c_{C}\left(W_{A B / X}+W_{B A / X}+W_{A B}^{R}\right) v_{A B_{\perp}^{+} \bar{C}} \\
& +2 c_{C} \sum_{\sigma}\left(W_{B A / \sigma} v_{A \sigma_{\perp}^{0} \bar{C}}-W_{A B / \sigma} v_{B \sigma_{\perp}^{0} \bar{C}}\right) \\
& \frac{d K_{i j}^{A B_{\perp}^{0} C}}{d t}=-4 c_{C}\left(W_{A B / X}+W_{B A / X}+W_{A B}^{R}\right) v_{A B_{\perp}^{0} \bar{C}} \\
& +c_{C} \sum_{\sigma}\left(W_{B A / \sigma}\left(v_{A \sigma_{\perp}^{+} \bar{C}}+v_{A \sigma_{\perp}^{0} \bar{C}}\right)+W_{A B / \sigma}\left(v_{B \sigma_{\perp}^{+} \bar{C}}+v_{B \sigma_{\perp}^{0} \bar{C}}\right)\right) \\
& +c_{C} W_{A B}^{R}\left(v_{A B_{\|}^{+} \bar{C}}+v_{B A_{\|}^{+} \bar{C}}+v_{A B_{\|}^{0} \bar{C}}+v_{B A_{\|}^{0} \bar{C}}\right) .
\end{aligned}
$$

It is worth noting that if the rotation frequencies $w_{A B}^{R}$ are equal to zero, combining those two kinetic equations leads to $v_{A B_{\perp}^{+} \bar{C}}=v_{A B_{\perp}^{0} \bar{C}}=0$. As a consequence, as long as the rotation is not taken into account, it is sufficient to deal with the effective interactions between a dumbbell and a nn target site.

\subsection{First shell approximation}

Even if the rotation is discarded, the resulting system of 17 equations in a binary alloy cannot be solved analytically. However, as mentionned in the above section, the system can reduce to only http://mc.manuscriptcentral.com/pm-pml 
one equation if we restrict the effective Hamiltonian to effective interactions inside the dumbbell. It is then sufficient to set to zero the one-point moment, so that only atoms in nn position with respect to the dumbbell are involved in the calculation of the transport coefficients : we will call the resulting model the first shell approximation, the second shell approximation consisting in solving the one-point and two-point kinetic equations. One obtains the expression of the effective field $h_{A B}$ as :

$$
h_{A B}=\frac{W_{B A / X}\left(\mu_{j+}^{A}-\mu_{i}^{A}\right)-W_{A B / X}\left(\mu_{j+}^{B}-\mu_{i}^{B}\right)}{W_{B A / X}+W_{A B / X}+W_{A B}^{R}} .
$$

We recognize in the denominator the average jump frequency of a mixed dumbbell $A B$, and define $W_{A B} \equiv W_{B A / X}+W_{A B / X}+W_{A B}^{R}$. The average jump frequency of the dumbbell is in fact $4 W_{A B}$, due to the four target sites for each atom and to the four possible rotations. Under the assumption of a limited effective Hamiltonian, we can express simply the correlation coefficients in a binary concentrated FCC alloy :

$$
\begin{aligned}
f_{A A} & =1-\frac{W_{A B / X}^{2}}{W_{A} W_{A B}} \\
f_{A B}^{(A)} & =\frac{W_{A B / X} W_{B A / X}}{W_{A} W_{A B}}
\end{aligned}
$$

and the coefficients $f_{B B}$ and $f_{B A}^{(B)}$ are obtained by inverting $A$ and $B$ in the above expressions.

In these conditions, it is also possible to give an analytical expression of the tracer correlation coefficient $f_{A}$ by considering a ternary system $A B A *$ infinitely dilute in the tracer element $A *$. The coefficient $f_{A}$ is then the limit of $f_{A * A *}$ and is given as :

$$
f_{A}=1-\frac{W_{A A / X}^{2}}{W_{A} W_{A A}}-\frac{W_{B A / X}^{2}}{W_{A} W_{A B}}
$$

This expression gives very simple results for the correlation factor of a dilute tracer in a pure matrix of element $A$ :

$$
\begin{aligned}
& f_{0}=f_{A}=1-\frac{w_{A A / A}}{2 w_{A A / A}+w_{A A}^{R}} \\
& \underset{\text { http://mc.manuscipiptedentral.com/pm-pml }}{f_{B}}
\end{aligned}
$$


In the dilute limit, $W_{A B / X}$ and $W_{B}$ reduce to $c_{A B} w_{A B / A}$ while $W_{A B}$ is $c_{A B}\left(w_{A B / A}+w_{B A / A}+w_{R}\right)$. If one assumes that the mixed dumbbell can neither dissociate $\left(w_{B A / A}=0\right)$ nor rotate $\left(w_{A B}^{R}=0\right)$, the coefficient $f_{B}$ is zero : the first-shell approximation of the SCMF is then sufficient to predict the well-known 'caging effect' in FCC crystals.

\section{BCC concentrated alloy}

\subsection{Geometry of the dumbbell}

In most BCC alloys, the most stable configuration of the dumbbell is along the $\langle 110\rangle$ direction, although the $\langle 111\rangle$ direction is also possible for mixed dumbbells in alloys [27, 21, 22, 23]. This study will be restricted to the first case, which has also been the most extensively studied up to now. In this system, four jump mechanisms have been used by Bocquet [28] : an on-site rotation $R$ of $60^{\circ}$ to another $\langle 110\rangle$ direction with four possible final orientations; a translation $T$ towards a nn target site ; a translation and rotation of $60^{\circ}$ towards the same $n n$ target site (RT) with two equivalent final orientations ; a translation to a second neighbour site followed by a rotation of $90^{\circ}$ (RT2). As for the last mechanism, we expect that a correct treatment with the SCMF theory would involve at least the use of second-neighbour effective interactions, which would considerably increase the complexity of the calculation. As a consequence, we will discard this mechanism and focus on the three others.

Target sites as a function of the orientation of the dumbbell are indicated on figure 2 : jumps $A B \rightarrow C$ are permitted for two sites $j+$, and $B A \rightarrow C$ for two sites $j-$ if $A B$ is directed towards the positive $X$ direction. Jumps $A B \rightarrow C$ and $B A \rightarrow C$ are permitted for one site $j+$ and one site $j$ - each if $A B$ is perpendicular to the $X$ direction.

In terms of jump frequencies, we differentiate the $\mathrm{T}$ and $\mathrm{RT}$ mechanisms by introducing a factor $\tau$ as :

$$
w_{A B / C}^{T}=\tau w_{A B / C}^{R T}
$$


The limit $\tau=0$ is equivalent to neglecting the simple translation, and $\tau=1$ sets a degeneracy between the three possible nn jumps. In real systems, this factor may depend on the chemical species involved in the jump of the defect. However, in a concentrated alloy it is probable that this effect would be averaged due to the high number of possible configurations. Consequently, we will restrict ourselves to a constant, and species independent, factor.

Concentrations $c_{A B}$ as well as generalized jump frequencies $W_{A B / C}, W_{A B / X}, W_{A B}^{R}$ and $W_{A}$ are defined as in section 3.1, except for Equation (29) which becomes :

$$
\sum_{A, B} c_{A B}=\frac{c_{I}}{6}
$$

because of the six possible orientations, instead of three in the FCC structure.

Throughout this section, we will derive the same equations as in the case of a FCC crystal : nevertheless, we found that the greater geometrical complexity deserved a complete description of the set of equations. As a consequence, we will just emphasize the differences between both structures and try not to repeat all explanations already given in the preceeding section.

\subsection{Expression of the flux}

We first express the flux of atoms $A$ by applying Equation (20) to our set of frequencies in the BCC structure :

$$
\begin{aligned}
J_{i \rightarrow s}^{A}= & -3(2+\tau) W_{A}\left(\mu_{s}^{A}-\mu_{i}^{A}\right) \\
& -(2+\tau) \sum_{\sigma, \sigma^{\prime}} c_{\sigma A} c_{\sigma^{\prime}}\left(2 h^{\sigma A}-2 v_{\sigma A_{\|}^{+} \overline{\sigma^{\prime}}}-v_{\sigma A_{\|}^{0} \overline{\sigma^{\prime}}}\right. \\
& \left.-2 h^{A \sigma^{\prime}}+2 v_{\sigma^{\prime} A_{\|}^{+} \bar{\sigma}}+v_{\sigma^{\prime} A_{\|}^{0} \bar{\sigma}}\right) w_{\sigma A / \sigma^{\prime}} \\
= & -3(2+\tau) W_{A}\left(\mu_{s}^{A}-\mu_{i}^{A}\right) \\
& -2(2+\tau) \sum_{\sigma, \sigma^{\prime}} c_{\sigma A} c_{\sigma^{\prime}}\left(2 h^{\sigma A}-2 v_{\sigma A_{\|}^{+} \overline{\sigma^{\prime}}}-v_{\sigma A_{\|}^{0} \overline{\sigma^{\prime}}}\right) w_{\sigma A / \sigma^{\prime}}
\end{aligned}
$$


In the first line, the factor $3(2+\tau)$ arises from the fact that on site $i$, three possible configurations of a dumbbell $\sigma A$ can jump toward site $j$, and each configuration can jump through two translationrotations or one simple translation. Within the three configurations, one is orthogonal to the $X$ direction, the other two being oriented positively relative to $X$.

\subsection{Kinetic moments}

We now express Equation (14) in terms of the simplified effective interactions in a BCC lattice. Considering the dumbbell $A B$ with a positive orientation on site $i$, let us call $j+$ a target site for the jump $A B / C$ and $j-$ a target site for the jump $B A / C$ :

$$
\begin{aligned}
\frac{d\left\langle n_{i}^{A B}\right\rangle}{d t}= & \left\langle n_{i}^{B} \sum_{\sigma} 2\left(n_{j-}^{\sigma A^{0}}+(1+\tau) n_{j-}^{\sigma A^{+}}\right) w_{\sigma A / B}\right. \\
& +n_{i}^{A} \sum_{\sigma} 2\left(n_{j+}^{B \sigma^{0}}+(1+\tau) n_{j+}^{B \sigma^{+}}\right) w_{\sigma B / A} \\
& -n_{i}^{A B^{+}} \sum_{\sigma}\left[2(2+\tau) n_{j-}^{\sigma} w_{B A / \sigma}+2(2+\tau) n_{j+}^{\sigma} w_{A B / \sigma}\right] \\
& \left.+\left(2 n_{i}^{A B^{0}}+2 n_{i}^{A B^{+}}-4 n_{i}^{A B^{+}}\right) w_{R}\right\rangle .
\end{aligned}
$$

The analysis of the first line is straightforward : it refers to the creation of a $A B^{+}$dumbbell on site $i$ (because the sign is positive) starting from a dumbbell $\sigma A$ on site $j-$. The factor 2 arises from the fact that two equivalent $j$ - sites are available. Focusing on one $j-$ site, the $A B^{+}$dumbbell can be created through a rotation-translation of a $\sigma A^{0}$ defect (first term), another rotation-translation of a $\sigma A^{+}$defect or a translation of another $\sigma A^{+}$defect. The latter two refer to two different orientations of the $\sigma A$ defect but they are equivalent in terms of effective interactions. 
We next derive it in terms of non-equilibrium quantities :

$$
\begin{aligned}
\frac{d\left\langle n_{i}^{A B}\right\rangle}{d t}= & 2(2+\tau) W_{A B / X}\left(\mu_{j+}^{B}-\mu_{i}^{B}\right)-2(2+\tau) W_{B A / X}\left(\mu_{j+}^{A}-\mu_{i}^{A}\right) \\
+ & \sum_{\sigma}\left[2 W _ { B A / \sigma } \left[(2+\tau)\left(v_{B A_{\|}^{+} \bar{\sigma}}+h_{A B}\right)\right.\right. \\
+ & \left.(1+\tau)\left(v_{\sigma A_{\|}^{+} \bar{B}}+h_{A \sigma}\right)+v_{\sigma A_{\|}^{0} \bar{B}}\right] \\
- & 2 W_{A B / \sigma}\left[(2+\tau)\left(v_{A B_{\|}^{+} \bar{\sigma}}-h_{A B}\right)\right. \\
& \left.\left.+(1+\tau)\left(v_{\sigma B_{\|}^{+} \bar{A}}-h_{\sigma B}\right)+v_{\sigma B_{\|}^{0} \bar{A}}\right]\right] \\
+ & 2 W_{A B}^{R} h_{A B}
\end{aligned}
$$

We give the expression of two more 2-point moments over the four possible to illustrate particular points of the calculation. The other two are formally very close to the latters, and can be found in Appendix D :

$$
\begin{aligned}
\frac{d\left\langle n_{i j}^{A B_{\|}^{0} C}\right\rangle}{d t}= & (2+\tau)\left(W_{A B / C}-W_{A B / X}\right)\left(\mu_{j+}^{B}-\mu_{i}^{B}\right) \\
& \left.+W_{A B / C}\left[2 h^{C B}-\left(2 v_{C B_{\|}^{+} \bar{A}}+\tau v_{C B_{\|}^{0} \bar{A}}\right)-(2+\tau) v_{A B_{\|}^{0} \bar{C}}\right)\right] \\
& +\sum_{\sigma} c_{C} W_{A B / \sigma}\left[-2 h^{\sigma B}-\left(2 v_{\sigma B_{\|}^{+} \bar{A}}+\tau v_{\sigma B_{\|}^{0} \bar{A}}+(2+\tau) v_{A B_{\|}^{0} \bar{\sigma}}\right)\right. \\
& \left.-(2+\tau) v_{A B_{\|}^{0} \bar{C}}\right]-\sum_{\sigma} 2(2+\tau) c_{C} W_{B A / \sigma} v_{A B_{\|}^{0} \bar{C}} \\
& +\left(2 v_{A B_{\|}^{+} \bar{C}}+2 v_{A B_{\perp}^{+} \bar{C}}-4 v_{A B_{\|}^{0} \bar{C}}\right) c_{C} W_{A B}^{R} .
\end{aligned}
$$

$$
\begin{aligned}
\frac{d K_{i j}^{A B_{\perp}^{+} C}}{d t}= & \left(2(2+\tau)\left(W_{A B / X}+W_{B A / X}\right)+3 W_{A B}^{R}\right) c_{C} v_{A B_{\perp}^{+} \bar{C}} \\
& -\left(v_{A B_{\|}^{+} \bar{C}}+v_{A B_{\perp}^{0} \bar{C}}+v_{B A_{\|}^{0} \bar{C}}\right) c_{C} W_{A B}^{R} .
\end{aligned}
$$

As in the FCC structure, if the rotation frequencies $w_{A B}^{R}$ are equal to zero, the kinetic equation reduces to $v_{A B_{\perp}^{+} \bar{C}}=0$, so that only effective interactions between a dumbbell and a target site need to be considered. 


\subsection{First shell approximation}

Eventually, we present the only analytical results achievable by this method, with the condition that effective interactions between a dumbbell and a substitutional atom are set to zero. It is then possible to express $h_{A B}$ as :

$$
h_{A B}=\frac{(2+\tau) W_{B A / X}\left(\mu_{j+}^{A}-\mu_{i}^{A}\right)-(2+\tau) W_{A B / X}\left(\mu_{j+}^{B}-\mu_{i}^{B}\right)}{(2+\tau)\left(W_{B A / X}+W_{A B / X}\right)+W_{A B}^{R}+(1+\tau)\left(W_{B A / B}+W_{A B / A}\right)} .
$$

We recognize in the denominator a mean frequency of the mixed dumbbell $W_{A B} \equiv(2+\tau)\left(W_{B A / X}+\right.$ $\left.W_{A B / X}\right)+W_{A B}^{R}$. As in the FCC case, we note that the total jump frequency of a dumbbell is not exactly $W_{A B}$ but $2 W_{A B}+2 W_{A B}^{R}$ : the first factor 2 is due to the presence of two target sites for each atom of the dumbbell in the BCC structure. On the other hand, even if four rotations are possible for a $A B^{+}$dumbbell, two among them lead to an equivalent $A B^{+}$configuration and are therefore not accounted for in the correlation effects. Furthermore, contrary to the FCC structure, the denominator does not consist only in the mean frequency $W_{A B}$. By analogy with the vacancy case, we call the other term $2 W^{\prime} \equiv(1+\tau)\left(W_{B A / B}+W_{A B / A}\right)$. Under the assumption of a limited effective Hamiltonian, we can express simply the correlation coefficients in a binary concentrated BCC alloy :

$$
\begin{aligned}
f_{A A} & =1-\frac{(2+\tau) W_{A B / X}^{2} / W_{A}}{W_{A B}+2 W^{\prime}} \\
f_{A B}^{(A)} & =\frac{(2+\tau) W_{A B / X} W_{B A / X} / W_{A}}{W_{A B}+2 W^{\prime}}
\end{aligned}
$$

and the coefficients $f_{B B}$ and $f_{B A}^{(B)}$ are obtained by inverting $A$ and $B$ in the above expressions. In these conditions, it is also possible to give an analytical expression of the tracer correlation coefficient $f_{A}$ in the binary alloy by considering a ternary system $A B A *$ infinitely dilute in the tracer element $A *$. The coefficient $f_{A}$ is then the limit of $f_{A * A *}$ and is given as :

$$
f_{A}=1-\frac{4(2+\tau)}{3 W_{A}} \times \frac{W_{A A / X}^{2} \tilde{W}_{A B} W_{B A / X}^{2} \tilde{W}_{A A}-4 W_{A A / X} W_{B A / X} W_{B A / A}}{\tilde{W}_{A A} \tilde{W}_{A B}-4 W_{A R+1 / p h l}^{2}}
$$


where $\tilde{W}_{i j}$ is a compact jump frequency defined as :

$$
\tilde{W}_{A B} \equiv(2+\tau)\left(W_{A B / X}+W_{B A / X}\right)+2 W_{A B}^{R}+(1+\tau) W_{A B / A}
$$

It is straightforward to deduce that in this case no caging effect is possible in a dilute alloy : this result is consistent as, in a BCC dilute alloy, a mixed dumbbell can migrate on long distances without dissociating, which is not the case in a FCC dilute alloy.

\section{Discussion}

In the two preceeding sections two approximations of the SCMF theory are presented for each cristallographic structure : the first shell approximation consists in a very simple analytic formulation of the transport coefficients, whereas one expects that a better accuracy will be achieved by the second shell approximation, which consists in solving a system of $n(n-1) / 2+4 n^{3}$ equations in a $n$-component system. Although the treatment of both structures is very similar within the framework of the SCMF formalism, it appears that only the FCC structure had yet been addressed successfully by other theories in the case of a concentrated alloy. In a first part, we compare the results of all models in a concentrated FCC alloy, and test the SCMF theory against available Monte Carlo simulations in a BCC alloy in a second part : simple relations between the four collective correlation coefficients are specifically examined. A last part is devoted to the incidence of the jump frequency model on the convergence of the SCMF theory.

\subsection{Comparison with existing models in FCC alloys}

Existing formalisms to calculate the transport coefficients in concentrated FCC alloys are due to Bocquet [9] and Chaturvedi and Allnatt $[12,13]$; they will be compared to both approximations of the SCMF theory on the basis of Monte Carlo simulations made by Bocquet [9].

Those simulations involve only two jump frequencies $w_{L}$ and $w_{R}$ (with $w_{L}<w_{R}$ ), and dumbbells http://mc.manuscriptcentral.com/pm-pml 
are allowed different stabilities as a function of their composition. Five sets of frequencies are possible as a function of the relative stabilities of $A A, A B$ and $B B$ in a binary alloy. If we denote $A A>A B$ the fact that $A A$ is more stable than $A B$, the five models are :

- Model 1, $B B>A B>A A, \quad w_{B B / A}=w_{L}, w_{B A / A}=w_{L}$;

- Model 2, $B B=A B>A A, \quad w_{B A / A}=w_{L}$;

- Model 3, $B B<A B>A A, \quad w_{B A / A}=w_{L}, w_{A B / B}=w_{L}$;

- Model 4, $B B>A B<A A, \quad w_{B B / A}=w_{L}, w_{A A / B}=w_{L}$;

- Model 5, $B B>A B=A A, \quad w_{A B / B}=w_{L}$,

all other jump frequencies being equal to $w_{R}$. Furthermore, rotation was discarded in all simulations.

Figures 4 and 5 show the results of all four theories in terms of the correlation coefficients $f_{i j}^{(i)}$ for the frequency models 1, 2, 3 and 5 for which the results of the model of Chaturvedi and Allnatt were available. As was already noted by Bocquet and Chaturvedi and Allnatt, the accuracy of all theories is strongly dependent on the chosen frequency model and on the correlation coefficient itself. In terms of relative accuracy of the competing models, no simple conclusion can be drawn except for the fact that the second shell approximation of the SCMF theory is more accurate than the first shell approximation, as expected. Apart from this simple result, we see for example that the SCMF is roughly more accurate than both others for the cross coefficient $f_{A B}^{(A)}$. As for $f_{B B}$, all three theories (the first shell approximation being discarded) present a comparable accuracy, excepted for the frequency models 1 and 5 where Bocquet's effective field theory is clearly more efficient. In models 2 and 3, it appears even sufficient to use the convenient first shell approximation to reach a satisfying value of all correlation coefficients. The same comments can be done concerning the tracer correlation factors, which are plotted on figure 6 for the same frequency models. http://mc.manuscriptcentral.com/pm-pml 
One can generally notice that the first shell approximation of the SCMF overestimates the correlation coefficients, while the formalism of Chaturvedi always underestimates those coefficients, when compared to Monte Carlo simulations.

[ Insert Figure 4 about here ]

[ Insert Figure 5 about here ]

[ Insert Figure 6 about here ]

Previous work on the vacancy case [3] have already emphasized the parallel between the SCMF theory and the random lattice gas approach by Okamura and Allnatt [29] or Moleko, Allnatt and Allnatt [2], which use the same formalism as the theory of Chaturvedi and Allnatt. Although the SCMF focuses on non-equilibrium systems whereas the random lattice gas formalism deals with equilibrium fluctuations, the kinetic equations are of the same nature in both theories. In the interstitial case, Equations (14) and (15) correspond to Equations (15) and (16) in ref. [12]. However, Chaturvedi and Allnatt had to take into account all 2-point moments whatever the distance between both points in order to carry out a Fourier transformation, which considerably enhanced the geometrical complexity of the calculation. In the present version of the SCMF, only nearest-neighbour two-point moments are accounted for. In the vacancy case, such an approximation did not have any important effect on the obtained correlation factors [4]. We can see on figures 4,5 and 6 that the equivalent approximation for the interstitials case (second shell approximation) is equally convenient for the interstitial mechanism and leads to a simpler calculation than the model of Chaturvedi and Allnatt. Furthermore, the equality $L_{A B}=L_{B A}$ is always fullfilled by the SCMF theory, whereas it was not the case in Chaturvedi and Allnatt's formalism.

On the second hand, the complexity of Bocquet's effective field theory is comparable to the SCMF one, and the former is expected to be superior to the present SCMF version in strongly correlated systems. Namely, the prediction of extreme correlation effects like a percolation threshold by SCMF http://mc.manuscriptcentral.com/pm-pml 
requires to account for $n$-point moments with $n \gg 2$ or to introduce a decoupling scheme in the kinetic equations, as was the case for the vacancy mechanism [3]. This scheme, formally parallel to the one introduced by Moleko, Allnatt and Allnatt [2], was comparable to the effective field theory of Manning [1] when applied to a binary alloy, but proved to be more efficient in presence of a third component. In conclusion, in systems presenting limited frequency ratios, the SCMF seems to be a reasonable alternative to Bocquet's formalism for the calculation of the transport coefficients, and appears superior to the formalism of Chaturvedi and Allnatt, the SCMF theory combining a comparable accuracy with a greater simplicity.

\subsection{Comparison with Monte Carlo simulations in a BCC alloy}

One direct advantage of the SCMF theory is its ability to treat the BCC concentrated alloy. In the same spirit as in FCC crystals, Bocquet [10] failed to find a satisfactory effective field approximation to calculate the correlation coefficients, and attributed his difficulties to the possibility for a dumbbell to jump towards a same target site in two or three different jumps with two or three different final orientations. Nevertheless, Monte Carlo simulations were performed in two-frequency systems [10, 11] in the same manner as in the FCC structure [9]. On the other hand, Sharma et al. [14] attempted to adapt the formalism of Chaturvedi and Allnatt to the BCC concentrated alloy but could not complete the calculation because of the geometrical complexity of their formalism. However, they provided with a relation between the four collective correlation coefficients, proving that only one coefficient out of four may be independent under some assumptions. Monte Carlo calculations by Belova and Murch were presented to support their result. Unfortunately, their simulations include the RT2 mechanism, which was not treated by this version of the SCMF. Consequently, we will compare the SCMF results to the Monte Carlo simulations by Bocquet $[10,11]$.

[ Insert Figure 7 about here ]

http://mc.manuscriptcentral.com/pm-pml 
In these particular Monte Carlo simulations [11], simple translation is accounted for with the ratio $\tau=1$ and the rotation frequencies of the dumbbells are set to zero. For jump ratios $w_{A} / w_{B}$ equal to 2 and 10, one can see that the first shell approximation of the SCMF already gives a very satisfying value of the correlation coefficients. Only the factor $f_{B A}^{(B)}$ is not exactly predicted for a jump frequency ratio of 10 , but once again the second shell approximation appears sufficient to achieve a quantitative agreement with the simulations. It is to notice that, with such a set of jump frequencies, all dumbbells have the same stability, which was not the case in figures 7(a) and 7(b).

As in the FCC case, treating alloys with high frequency ratios would certainly require to integrate a decoupling scheme to the present version of the SCMF. However, building such a scheme in the BCC structure would probably present additional difficulties due to the degeneracy of the jump mechanisms $\mathrm{T}$ and RT, which let the dumbbell jump from one given site to the same target site with several different final orientations. For example, in the first shell approximation, this degenerency may http://mc.manuscriptcentral.com/pm-pml 
be the cause of the appearance of the frequency $W^{\prime}$ in the expression of the correlation coefficients. This frequency is the only notable difference between the expressions in the BCC and FCC structures (for which it is absent), although a satisfying interpretation is still missing.

\subsection{Sharma et al. relations}

As was mentioned in the preceeding paragraph, the main result of the work of Sharma et al. [14] consisted in a set of relations between the correlation coefficients in a concentrated binary BCC alloy. Using the notations of the present paper, those relations are expressed as :

$$
\begin{aligned}
f_{B B} & =1-\left(\frac{W_{A B / X}}{W_{B A / X}}\right)^{2} \frac{W_{A}}{W_{B}}\left(1-f_{A A}\right) \\
f_{A B}^{(A)} & =\frac{W_{A B / X}}{W_{B A / X}}\left(1-f_{A A}\right) \\
f_{A B}^{(B)} & =\frac{W_{A B / X}}{W_{B A / X}} \times \frac{W_{A}}{W_{B}}\left(1-f_{A A}\right) .
\end{aligned}
$$

From the expressions of the correlation coefficients in Equations (56) and (57), it is very simple to show that the above relations are always fulfilled by the first shell approximation of the SCMF in a BCC alloy. We also observed a numerical agreement of the second shell approximation with the Sharma et al. relations, although an analytical proof is not available. A more interesting feature is that the same is true for the FCC structure (see Equations (43) and (44) for the first shell approximation). This unexpected similarity supports the hypothesis of Sharma et al., that these relations may be of general validity for the dumbbell mechanism in any concentrated random alloy, as the analogous relations of Moleko and Allnatt [30] for the vacancy mechanism.

It is also worth pointing out that the Sharma et al. relations are fulfilled at the same time by both approximations of the SCMF theory, as well as by the formalism of Chaturvedi, which should correspond to the SCMF formalism including infinite-range 
pair effective interactions.. The situation is the same with the Moleko and Allnatt relations for the vacancy mechanism, which are fulfilled by the SCMF formalism whatever the range of pair effective interactions $[4,3]$. Considering eventually the good agreement of Equations (61)-(63) with Monte Carlo simulations [14], the authors highlight the importance of such relations for further developments on the topic of diffusion by the dumbbell mechanism.

\subsection{Incidence of the jump frequency model}

In this paragraph we propose an interpretation of the correlation effects obtained by the SCMF theory as the probability of a backward displacement after one given jump : such an interpretation was extensively used for the vacancy mechanism $[1,3]$, and was used among others by Bocquet [26] for the dumbbell mechanism. Let us consider a FCC alloy, in which each atom of a dumbbell has four target sites, and in this alloy a particular jump $A B \rightarrow C$. After this jump, the resulting $B C$ dumbbell can either operate a reverse jump with the frequency $w_{C B / A}$ or any other jump with a total escape frequency. In the SCMF formalism, the first shell and second shell approximations consist in approximating those two frequencies.

The competition between a single return frequency and a mean escape frequency is only taken into account by the SCMF theory for the second shell aproximation (see Equation (38) and the subsequent remark) : in this approximation, the return frequency is

$$
w_{\text {ret }}^{(2)}=w_{C B / A}
$$

and the escape frequency becomes

$$
H^{(2)}=\sum_{X} 4 w_{B C / X} c_{X}+3 w_{C B / X} c_{X}
$$

as if atoms $A, B$ and $C$ were embedded in an average medium. In the first shell approximation, the backward frequency $w_{C B / A}$ does not appear in the formalism. An interpretation of this absence is 
that the defect $B C$ may 'forget' the nature of atom $A$ after the first jump : the return and escape frequencies would then have the values :

$$
\begin{aligned}
w_{\text {ret }}^{(1)} & =\sum_{X} w_{C B / X} c_{X} \\
H^{(1)} & =\sum_{X} 4 w_{B C / X} c_{X}+3 w_{C B / X} c_{X} .
\end{aligned}
$$

One way to consider the influence of the jump frequency model on the accuracy of both approximations is then to study the incidence of the frequency model in terms of return frequency, as the escape frequency is the same for both approximations. If the jump frequencies depend only on the jumping species, the return frequency is $w_{B}$ in both approximations : as a consequence, the difference between first and second second shell may not be important, which agrees with the results shown on figure 7 for jump frequency ratios of 2 and 10. Nevertheless, this interpretation in terms of return and escape frequencies is only approximate, and it is to expect that, for higher jump frequency ratios, the difference between both approximations should increase. On the other hand, if the dumbbells are allowed different stabilities, e.g. if $B C$ is more stable than $A B$, the return frequency in second shell will be lower and more accurate than the return frequency in first shell :

$$
w_{C B / A}<\sum_{X} w_{C B / X} c_{X}
$$

As a consequence, the second shell approximation should be more efficient in terms of correlation effects than the first shell approximation. In conclusion, for a given jump frequency ratio, the contribution of the second-shell approximation with respect to the first-shell will be more important in presence of a difference of stabilities between dumbbells. The first-shell approximation will then be prefered for systems with limited jump frequency ratios and without important differences of dumbbell binding energies. Nevertheless, a more careful analysis is required to make a parallel between the approximations of the SCMF theory and the actual paths of the defect, as was done recently for the vacancy mechanism [3] : in particular, the different efficiency of the SCMF method http://mc.manuscriptcentral.com/pm-pml 
as a function of the different models proposed by Bocquet is still not clear.

\section{Conclusion}

We presented a new self-consistent mean field (SCMF) theory to calculate the transport coefficients in alloys with interstitial mechanism. This theory is an adaptation of the recent SCMF model successfully proposed for the vacancy mechanism, and is based on a thermodynamic-like description of a non-equilibrium partition function and classical kinetic equations. Under judicious assumptions, this theory provides with simple analytic expressions of the transport coefficients (first shell approximation) or a more complex calculation (second shell approximation). The results are found as accurate as competing theories by Bocquet [9] or Chaturvedi and Allnatt [12, 13] in FCC non-interacting concentrated alloys with limited jump frequency ratios, although the SCMF theory seems easier to generalize to more complex systems. In BCC non-interacting concentrated alloy, the SCMF theory is the first yet to complete the calculation of the transport coefficients, and exhibits a good agreement with Monte Carlo simulations for jump frequency ratios equal to 10. Compared to the Chaturvedi and Allnatt $[12,13]$ formalism, the SCMF theory uses very similar kinetic equations although in a less complex framework : this consideration lets expect a good efficiency of the SCMF also in dilute alloys, which will be the subject of a coming article.

Generally speaking, the existence of an analytic expression of the correlation coefficients in a concentrated alloy in both structures is a valuable tool for the further developments of this theory and for the study of transport by interstitial mechanism. Simple relations between the correlation coefficients, introduced by Sharma et al. [14], were also supported by this work. Another important investigation could concern the role of the on-site rotation on correlation effects, which was discarded in the above Monte Carlo simulations : this particular mechanism is characteristic of the dumbbell mechanism and deserves in our opinion http://mc.manuscriptcentral.com/pm-pml 
a complete study. Next efforts will be devoted to extend the domain of validity of the SCMF in concentrated alloys to strongly correlated systems or to alloys with a more complete thermodynamic description, as was done recently for the vacancy case $[5,3]$.

\section{Acknowledgements}

The authors are thankful to J.L. Bocquet, A. Barbu, G. Martin, C. Hin and A.B. Lidiard for their valuable support and comments. Private communications from J.L. Bocquet were particularly appreciated. This work was funded by the joint program SMIRN (CEA, EDF, CNRS).

http://mc.manuscriptcentral.com/pm-pml 


\section{Appendix A : Linearization of the kinetic equations}

We detail above the linearization for one pair of terms in Equation (14), the procedure being similar for the other terms. We consider the quantity $\Delta_{\sigma A / B}^{\beta \alpha}$ which controls the transitions between the dumbbells $\sigma A_{\beta}$ on site $s$ and $A B_{\alpha}$ on site $i$ :

$$
\Delta_{\sigma A / B}^{\beta \alpha}=\left\langle n_{s}^{\sigma A_{\beta}} n_{i}^{B} \gamma_{s i}^{\beta \alpha} w_{\sigma A / B}^{\beta \alpha}-n_{i}^{A B_{\alpha}} n_{s}^{\sigma} \gamma_{i s}^{\bar{\alpha} \bar{\beta}} w_{B A / \sigma}^{\bar{\alpha} \bar{\beta}}\right\rangle
$$

We first apply Equation (5) to change this expression into an equilibrium average :

$$
\Delta_{\sigma A / B}^{\beta \alpha}=\left\langle n_{s}^{\sigma A_{\beta}} n_{i}^{B} \gamma_{s i}^{\beta \alpha} w_{\sigma A / B}^{\beta \alpha} P_{1}(\mathbf{n})-n_{i}^{A B_{\alpha}} n_{s}^{\sigma} \gamma_{i s}^{\bar{\alpha} \bar{\beta}} w_{B A / \sigma}^{\bar{\alpha} \bar{\beta}} P_{1}(\mathbf{n})\right\rangle^{(0)}
$$

where \langle\rangle$^{(0)}$ stands for an equilibrium average over all configurations weighted by $\hat{P}_{0}$.

The next step is to expand Equation (6) to the first order with respect to the non-equilibrium quantities $\beta \delta \mu_{i}^{A}(t)$ and $\beta \hat{h}(t)$. Those quantities are assumed to be close to zero as the system is close to equilibrium :

$$
\begin{aligned}
\Delta_{\sigma A / B}^{\beta \alpha}= & \left\langle n_{s}^{\sigma A_{\beta}} n_{i}^{B} \gamma_{s i}^{\beta \alpha} w_{\sigma A / B}^{\beta \alpha}[1+\beta(\delta \Omega(t)\right. \\
& \left.\left.+\delta \mu_{s}^{A}(t)+\delta \mu_{s}^{\sigma}(t)+\delta \mu_{i}^{B}(t)+\delta \mu_{s}^{I}(t)-\hat{h}_{s}^{\sigma A_{\beta}}(t)\right)\right] \\
& -n_{i}^{A B_{\alpha}} n_{s}^{\sigma} \gamma_{i s}^{\bar{\alpha} \bar{\beta}} w_{B A / \sigma}^{\bar{\alpha} \bar{\beta}}[1+\beta(\delta \Omega(t) \\
& \left.\left.\left.+\delta \mu_{i}^{A}(t)+\delta \mu_{i}^{B}(t)+\delta \mu_{s}^{\sigma}(t)+\delta \mu_{i}^{I}(t)-\hat{h}_{i}^{A B_{\alpha}}(t)\right)\right]\right\rangle^{(0)},
\end{aligned}
$$

where $\hat{h}_{i}^{A B_{\alpha}}$ is the partial derivative of $\hat{h}$ with respect to the occupation number $n_{i}^{A B_{\alpha}}$. In this expression we have omitted the terms of the form $\hat{h}_{i}^{A}$ where $A$ is a substitutional atom, because they are of a higher order in terms of the dumbbell concentration. Furthermore, the time dependence of the effective interactions will be dropped in the rest of the article, given that the kinetic equations are to be solved in steady state. Note that we use the property of the occupation numbers

$$
\text { http://mc.manu'scriptcentral.com/pm-pml }
$$


where $\delta_{A B}$ is the Kroenecker symbol, to derive the more general expression :

$$
\left\langle n_{i}^{A B_{\alpha}} \hat{h}\right\rangle^{(0)}=\left\langle n_{i}^{A B_{\alpha}} \hat{h}_{i}^{A B_{\alpha}}\right\rangle^{(0)}
$$

Eventually, we take advantage of the detailed balance (Equation (16)) to derive the expression :

$$
\Delta_{\sigma A / B}^{\beta \alpha}=\beta\left\langle n_{s}^{\sigma A_{\beta}} n_{i}^{B} \gamma_{s i}^{\beta \alpha} w_{\sigma A / B}^{\beta \alpha}\left[\left(\delta \mu_{s}^{A}+\delta \mu_{s}^{I}\right)-\left(\delta \mu_{i}^{A}+\delta \mu_{i}^{I}\right)-\hat{h}_{s}^{\sigma A_{\beta}}+\hat{h}_{i}^{A B_{\alpha}}\right]\right\rangle^{(0)}
$$

We note that the difference of the non-equilibrium part of the chemical potentials $\left(\delta \mu_{s}^{A}-\delta \mu_{i}^{A}\right)$ is equal to the difference of the total chemical potentials $\left(\mu_{s}^{A}-\mu_{i}^{A}\right)$ since the chemical potential at equilibrium is uniform. Furthermore, in all kinetic equations of n-point moments, the chemical potentials of the atomic species will always be grouped with the one of defects in the form $\mu_{i}^{A}+\mu_{i}^{I}$. Subsequently, we will replace the latter expression by $\mu_{i}^{A}$, given that the chemical potential of defects will not appear separately in the kinetic equations. The simple final form of $\Delta_{\sigma A / B}^{\beta \alpha}$ is :

$$
\Delta_{\sigma A / B}^{\beta \alpha}=\beta\left\langle n_{s}^{\sigma A_{\beta}} n_{i}^{B} \gamma_{s i}^{\beta \alpha} w_{\sigma A / B}^{\beta \alpha}\left(\mu_{s}^{A}-\mu_{i}^{A}-\hat{h}_{s}^{\sigma A_{\beta}}+\hat{h}_{i}^{A B_{\alpha}}\right)\right\rangle^{(0)}
$$

\section{Appendix B : Equilibrium concentrations of the different dumbbell configurations}

Several methods are available to calculate the equilibrium concentration of the different species of dumbbells in a multicomponent alloy. In the case of a non-interacting alloy, it may be most convenient to simply solve the equations of the detailed balance, of the type of Equation (16). We present here a more general calculation within the grand canonical ensemble, which will be easier to generalize to more complex systems. The principle of this method is to minimize the grand potential of the system. It is expressed as :

$$
\begin{aligned}
\Omega= & \sum_{A, B, \alpha}\left(\epsilon_{A B} x_{A B_{\alpha}}+k_{B} T L\left(x_{A B_{\alpha}}\right)\right)+\sum_{A} k_{B} T L\left(x_{A}\right) \\
& +\sum_{A} \lambda_{A}\left(x_{A}+2 \sum_{\alpha} x_{A A \alpha}+\sum_{\alpha, B \neq A} x_{A B_{\alpha}}-c_{A}\right) \\
& +\lambda_{I}\left(\sum_{\text {http.,P/:mc.manuscriptcentral.com/pm-pml }} x_{A B_{\alpha}}-c_{I}\right),
\end{aligned}
$$


where notations are defined as follows : $x_{A}$ and $x_{A B_{\alpha}}$ are the unknown concentrations of species $A$ and dumbbells $A B_{\alpha}$ at equilibrium, $k_{B} T$ is the temperature multiplied by the Boltzmann constant, $L(x)=$ $x \ln x-x$ is the entropy term, $c_{A}$ and $c_{I}$ are the imposed value for the equilibrium concentration of species $A$ and the total concentration of dumbbells, $\epsilon_{A B}$ is the binding energy of the dumbbell $A B$, and the factors $\lambda$ are Lagrange operators used to calculate the $x$ values. Although the dumbbells are divided into different species, one must take one unique factor $\lambda_{I}$ for all dumbbells.

To reach those values, the first step is to minimize the energy of the system with respect to the $x$ terms :

$$
\frac{\partial \Omega}{\partial x}=0
$$

which gives rise to a relation between the $x$ and the Lagrange operators :

$$
\begin{aligned}
x_{A} & =\exp \left[-\frac{\lambda_{A}}{k_{B} T}\right] \\
x_{A B_{\alpha}} & =\exp \left[-\frac{\epsilon_{A B}+\lambda_{A}+\lambda_{B}+\lambda_{I}}{k_{B} T}\right] .
\end{aligned}
$$

In a last step, the Lagrange operators are calculated so as to satisfy the required concentrations. In the point approximation, this condition states simply :

$$
\begin{aligned}
x_{A} & =c_{A} \\
\sum_{\alpha, A, B} x_{A B_{\alpha}} & =c_{I},
\end{aligned}
$$

which leads to the concentrations of the different types of dumbbells. In a binary FCC alloy $A B$, the resulting concentrations of dumbbells are :

$$
\begin{aligned}
c_{A A} & =\frac{P_{A A}}{P_{A A}+P_{B B}+2} \times \frac{c_{I}}{3} \\
c_{B B} & =\frac{P_{B B}}{P_{A A}+P_{B B}+2} \times \frac{c_{I}}{3} \\
c_{A B} & =\frac{2}{P_{A A}+P_{B B}+2} \times \frac{c_{I}}{3},
\end{aligned}
$$


where the factors $P_{A A}$ and $P_{B B}$ are defined as:

$$
\begin{aligned}
P_{A A} & =\frac{c_{A}}{c_{B}} \exp \left(\frac{\epsilon_{A B}-\epsilon_{A A}}{k_{B} T}\right) \\
P_{B B} & =\frac{c_{B}}{c_{A}} \exp \left(\frac{\epsilon_{A B}-\epsilon_{B B}}{k_{B} T}\right) .
\end{aligned}
$$

It is worth noting that the same result could be achieved by solving the equations of the detailed balance (Equation (16)) taking advantage of the relation between the binding energies and the jump frequencies :

$$
\begin{aligned}
& \frac{w_{B B / A}}{w_{A B / B}}=\exp \left(\frac{\epsilon_{B B}-\epsilon_{A B}}{k_{B} T}\right) \\
& \frac{w_{A A / B}}{w_{B A / A}}=\exp \left(\frac{\epsilon_{A A}-\epsilon_{A B}}{k_{B} T}\right) .
\end{aligned}
$$

\section{Appendix C : Summation of effective interactions}

In this paragraph we detail the calculation of the expression

$$
S_{A B_{\|}^{+} C} \equiv\left\langle n_{i}^{A B_{\alpha}} n_{j}^{C} \hat{h}_{i}^{A B_{\alpha}}\right\rangle^{(0)}
$$

which appears in all kinetic equations. Here we consider that the effective interactions associated to the complex $\mathrm{ABC}$ is $v_{A B_{\|}^{+} C}$ in a $\mathrm{FCC}$ structure, the procedure being the same for other relative orientations as well as for the BCC structure. As shown in figure 1 , atom $C$ is situated on a ' $j+$ '-type site.

We start by recalling the absence of interactions involving substitutional atoms : as a consequence, it is straightforward to derive the equality :

$$
\begin{aligned}
\left\langle n_{i}^{A B_{\alpha}} n_{j}^{C} n_{k}^{D} \ldots\right\rangle^{(0)} & =\left\langle n_{i}^{A B_{\alpha}}\right\rangle^{(0)}\left\langle n_{j}^{C} \ldots\right\rangle^{(0)}\left\langle n_{k}^{D}\right\rangle^{(0)} \ldots \\
& =c_{A B} c_{C} c_{D} \ldots
\end{aligned}
$$

where the sites $i, j, \ldots$ are omitted because the equilibrium state of the system is uniform.

http://mc.manuscriptcentral.com/pm-pml 
In a second step, considering the form of the effective Hamiltonian (Equation (7)), we can express the derivative of $\hat{h}$ with respect to the occupation numbers as :

$$
\hat{h}_{i}^{A B_{\alpha}}=v_{A B}+\sum_{\sigma} 4\left(n_{j+}^{\sigma} v_{A B_{\|}^{+} \sigma}+n_{j 0}^{\sigma} v_{A B_{\perp}^{+} \sigma}-n_{j-}^{\sigma} v_{B A_{\|}^{+} \sigma}\right)
$$

Eventually, with help of Equation (72), we can then calculate the expression

$$
\begin{aligned}
S_{A B_{\|}^{+} C}= & c_{A B} c_{C}\left[v_{A B}+\right. \\
& \left.\left.\sum_{\sigma}\left(3 v_{A B_{\|}^{+} \sigma}+4 v_{A B_{\perp}^{+} \sigma}-4 v_{B A_{\|}^{+} \sigma}\right) c_{\sigma}\right)+v_{A B_{\|}^{+} C}\right] \\
= & c_{A B} c_{C}\left[v_{A B}+4 \sum_{\sigma}\left(v_{A B_{\|}^{+} \sigma}+v_{A B_{\perp}^{+} \sigma}+v_{B A_{\|}^{+} \sigma}\right) c_{\sigma}\right) \\
& \left.-\sum_{\sigma} v_{A B_{\|}^{+} \sigma_{\sigma}} c_{\sigma} v_{A B_{\|}^{+} C}\right] .
\end{aligned}
$$

Given the definitions of the compact notations (Equations (30) and (31)), this expression becomes :

$$
S_{A B_{\|}^{+} C}=c_{A B} c_{C}\left[h_{A B}-v_{A B_{\|}^{+} \bar{C}}\right]
$$

\section{Appendix D : Expression of the two-point moments}

All missing two-point moments are given here to allow the calculation of the transport coefficients.

FCC structure :

$$
\begin{aligned}
\frac{d\left\langle n_{i j}^{A B_{\|}^{0} C}\right\rangle}{d t}= & \left(W_{A B / C}-c_{C} W_{A B / X}\right)\left(\mu_{j+}^{B}-\mu_{i}^{B}\right) \\
& +\sum_{\sigma} W_{A B / \sigma} c_{C}\left(-h_{\sigma B}+v_{\sigma B_{\|}^{+} \bar{A}}+v_{A B_{\|}^{0} \bar{\sigma}}-v_{A B_{\|}^{0} \bar{C}}\right) \\
& +2 \sum_{\sigma} W_{A B / \sigma} c_{C}\left(v_{\sigma B_{\|}^{0} \bar{C}}-v_{A B_{\|}^{0} \bar{C}}\right) \\
& +W_{A B / C}\left(h_{C B}-v_{C B_{\|}^{+} \bar{A}}-v_{A B_{\|}^{0} \bar{C}}\right) \\
& +c_{C} W_{A B}^{R}\left(v_{A B_{\|}^{+} \bar{C}}+v_{B A_{\|}^{+} \bar{C}}+2 v_{A B_{\perp}^{0} \bar{C}}-4 v_{A B_{\|}^{0} \bar{C}}\right) W_{A B}^{R} .
\end{aligned}
$$


BCC structure :

$$
\begin{aligned}
& \frac{d\left\langle n_{i j}^{A B_{\|}^{+} C}\right\rangle}{d t}=(2+\tau)\left(W_{A B / C}+c_{C} W_{A B / X}\right)\left(\mu_{j+}^{B}-\mu_{i}^{B}\right) \\
& -2(2+\tau) c_{C} W_{B A / X}\left(\mu_{i}^{A}-\mu_{j-}^{A}\right) \\
& +W_{A B / C}\left[(1+\tau) h_{C B}-\left((1+\tau) v_{C B_{\|}^{+}} \bar{A}+v_{C B_{\|}^{0} \bar{A}}\right)\right. \\
& \left.+(2+\tau)\left(h_{A B}-v_{A B_{\|}^{+} \bar{C}}\right)\right] \\
& +\sum_{\sigma} c_{C} W_{A B / \sigma}\left[(1+\tau) h_{\sigma B}-\left((1+\tau) v_{\sigma B_{\|}^{+} \bar{A}}+v_{\sigma B_{\|}^{0} \bar{A}}\right)\right. \\
& \left.-(2+\tau) v_{A B_{\|}^{+} \bar{\sigma}}+(2+\tau)\left(h_{A B}-v_{A B_{\|}^{+} \bar{C}}\right)\right] \\
& +2 \sum_{\sigma} c_{C} W_{B A / \sigma}\left[-(1+\tau) h_{\sigma A}+\left((1+\tau) v_{\sigma A_{\|}^{+} \bar{B}}+v_{\sigma A_{\|}^{0} \bar{B}}\right)\right. \\
& \left.+(2+\tau) v_{B A_{\|}^{+} \bar{\sigma}}+(2+\tau)\left(h_{A B}-v_{A B_{\|}^{+} \bar{C}}\right)\right] \\
& +\left(-2 h_{A B}+v_{A B_{\|}^{0} \bar{C}}+v_{A B_{\perp}^{0} \bar{C}}+v_{A B_{\perp}^{+} \bar{C}}-3 v_{A B_{\|}^{+} \bar{C}}\right) W_{A B}^{R} . \\
& \frac{d\left\langle n_{i j}^{A B_{\perp}^{0} C}\right\rangle}{d t}=-6(2+\tau) c_{C} W_{A B}^{R} v_{A B_{\perp}^{+} \bar{C}} \\
& +\left(v_{A B_{\|}^{+} \bar{C}}+v_{A B_{\perp}^{+} \bar{C}}+v_{B A_{\|}^{+} \bar{C}}+v_{B A_{\perp}^{+} \bar{C}}\right) c_{C} W_{A B}^{R} .
\end{aligned}
$$

http://mc.manuscriptcentral.com/pm-pml 


\section{References}

[1] J. R. Manning. Phys. Rev. B, 4:1111, 1971.

[2] L. K. Moleko, A. R. Allnatt, and E. L. Allnatt. Phil. Mag. A, 59:141, 1989.

[3] V. Barbe and M. Nastar. Phil. Mag., in press, 2006.

[4] M. Nastar, V. Yu. Dobretsov, and G. Martin. Phil. Mag. A, 80:155, 2000.

[5] M. Nastar. Phil. Mag., 85:3767, 2005.

[6] Z. Qin, A. R. Allnatt, and E. L. Allnatt. Phil. Mag. A, 71:296, 1995.

[7] H. Sato, T. Ishikawa, and R. Kikuchi. J. Phys. Chem. Solids, 46:1361, 1985.

[8] J. L. Bocquet. Acta Metall., 34:571, 1986.

[9] J. L. Bocquet. Res Mechanica, 22:1, 1987.

[10] J. L. Bocquet. Technical Report CEA-R-5531, 1990.

[11] J. L. Bocquet. private communication.

[12] D. K. Chaturvedi and A. R. Allnatt. Phil. Mag. A, 69:821, 1994.

[13] D. K. Chaturvedi and A. R. Allnatt. Phil. Mag. A, 70:657, 1994.

[14] S. Sharma, D. K. Chaturvedi, I. V. Belova, and G. E. Murch. Phil. Mag., 80:65, 2000.

[15] A. Barbu. Acta Met., 28:499, 1980.

[16] A. R. Allnatt, A. Barbu, A. D. Franklin, and A. B. Lidiard. Acta Met., 31:1307, 1983.

[17] A. R. Allnatt and A. B. Lidiard. Atomic Transport in Solids. Cambridge University Press, 1993. http://mc.manuscriptcentral.com/pm-pml 
[18] A. Barbu and A. B. Lidiard. Phil. Mag. A, 74:709, 1996.

[19] R. Singh and D. K. Chaturvedi. Phys. Rev. B, 48:16366, 1993.

[20] S. Sharma, D. K. Chaturvedi, I. V. Belova, and G. E. Murch. Phil. Mag., 81:431, 2001.

[21] K. H. Robrock. Mat. Sci. Forum, 15:537, 1987.

[22] C. C. Fu, F. Willaime, and P. Ordejón. Phys. Rev. Lett., 92:175503, 2004.

[23] C. Domain and C. S. Becquart. Phys. Rev. B, 71:214109, 2005.

[24] M. Nastar and E. Clouet. Phys. Chem. and Chem. Phys., 6:3611, 2000.

[25] V. G. Vaks, S. V. Beiden, and V. Yu. Dobretsov. JETP Lett., 61:68, 1995.

[26] J. L. Bocquet. Phil. Mag. A, 47:547, 1983.

[27] R. A. Johnson. J. Phys. F, 3:295, 1973.

[28] J. L. Bocquet. Phil. Mag. A, 63:157, 1991.

[29] Y. Okamura and A.R. Allnatt. Phil. Mag. A, 71:307, 1986.

[30] L. K. Moleko and A. R. Allnatt. Phil. Mag. A, 58:677, 1988.

http://mc.manuscriptcentral.com/pm-pml 
Figure 1 : Geometry of the $\langle 100\rangle$ dumbbell in the FCC structure in the case of a one-dimensional diffusion along the $X$ axis. The configurations to be taken into account are (a) : $A B^{0} ;(\mathrm{b})$ : $A B_{\|}^{0} C ;(\mathrm{c}): A B_{\perp}^{0} C ;(\mathrm{d}): A B^{+} ;(\mathrm{e}): A B_{\|}^{+} C ;(\mathrm{f}): A B_{\perp}^{+} C$. Four sites $j+$ and four sites $j-$ are present in this structure. Target sites for atom $B$ are sites S1, S2, S3 and S4 for the dumbbell $A B^{0}$, sites $\mathrm{S} 4, \mathrm{~S} 5, \mathrm{~S} 6, \mathrm{~S} 7$ for the dumbbell $A B^{+}$.

Figure 2 : Geometry of the $\langle 110\rangle$ dumbbell in the BCC structure in the case of a one-dimensional diffusion. The configurations to be taken into account are (a) : $A B^{0} ;$ (b) : $A B_{\|}^{0} C ;$ (c) : $A B_{\perp}^{0} C ;(\mathrm{d}): A B^{+} ;(\mathrm{e}): A B_{\|}^{+} C ;(\mathrm{f}): A B_{\perp}^{+} C$. Four sites $j+$ and four sites $j-$ are present in this structure. Target sites for atom $B$ are sites $\mathrm{S} 1$ and $\mathrm{S} 5$ for the dumbbell $A B^{0}$, sites $\mathrm{S} 7$ and S8 for the dumbbell $A B^{+}$.

Figure 3 : Illustration of the antisymmetry property of the effective interactions in the BCC structure. The effective interactions associated to the marked atoms are respectively : (a) $-v_{A B_{\|}^{0} C}$;
(b) $-v_{A B_{\|}^{+} C} ;$ (c) $-v_{A B_{\perp}^{+} C}$.

Figure 4 : Correlation coefficients in a $A B$ concentrated FCC alloy as a function of the concentration $\mathrm{C}(\mathrm{B})$ for the frequency models 1 (top) and 2 (bottom) with $w_{R} / w_{L}=10$. The full triangles stand for the Monte Carlo simulations by Bocquet [9], empty triangles for the theory of Chaturvedi and Allnatt [13], dashed lines for the theory of Bocquet [9]. The SCMF results are plotted in dotted lines (first shell approximation) and solid lines (second shell approximation).

Figure 5: Correlation coefficients in a $A B$ concentrated FCC alloy as a function of the concentration $\mathrm{C}(\mathrm{B})$ for the frequency models 3 (top) and 5 (bottom) with $w_{R} / w_{L}=10$. The full triangles stand for the Monte Carlo simulations by Bocquet [9], empty triangles for the theory of Chaturvedi and Allnatt [13], dashed lines for the theory of Bocquet [9]. The SCMF results are plotted in dotted lines (first shell approximation) and solid lines (second shell approximation). http://mc.manuscriptcentral.com/pm-pml 
Figure 6 : Tracer correlation coefficients in a $A B$ concentrated FCC alloy as a function of the concentration $\mathrm{C}(\mathrm{B})$ for several Bocquet's models with $w_{R} / w_{L}=10$. The full triangles stand for the Monte Carlo simulations by Bocquet [9], empty triangles for the theory of Chaturvedi and Allnatt [12], dashed lines for the theory of Bocquet [9]. The SCMF results are plotted in dotted lines (first shell approximation) and solid lines (second shell approximation). (a) : model $1 ;(\mathrm{b}):$ model $2 ;(\mathrm{c}): \operatorname{model} 3 ;(\mathrm{d}): \operatorname{model} 5$;

Figure 7 : Correlation coefficients in a $A B$ concentrated BCC alloy as a function of the concentration $\mathrm{C}(\mathrm{B})$ for the frequency model 5 (top) with $w_{R} / w_{L}=10$, and for equation (60) (bottom) with $w_{A} / w_{B}=10$. The full symbols stand for the Monte Carlo simulations by Bocquet $[10,11]$. The SCMF results are plotted in dotted lines (first shell approximation) and solid lines (second shell approximation).

http://mc.manuscriptcentral.com/pm-pml 


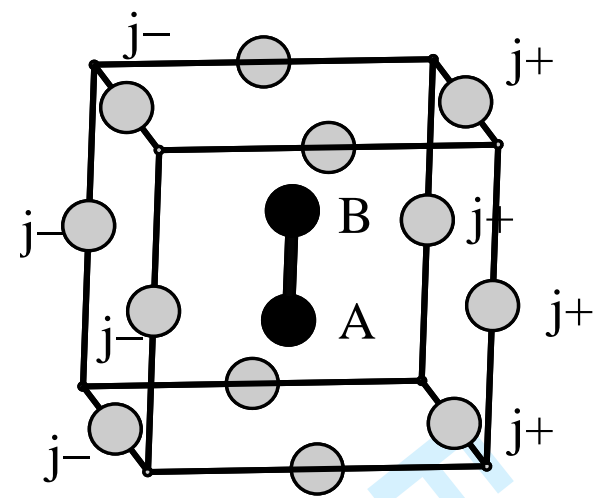

(a)

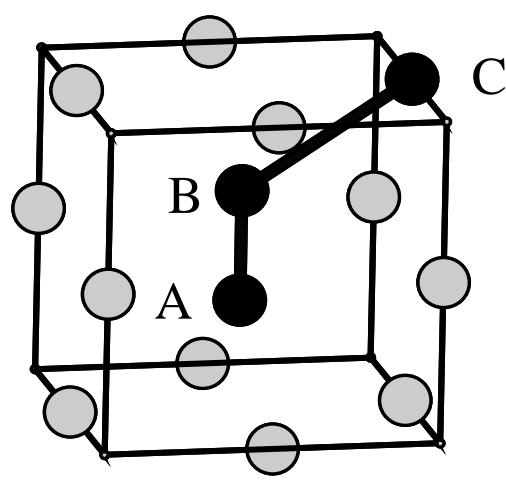

(b)

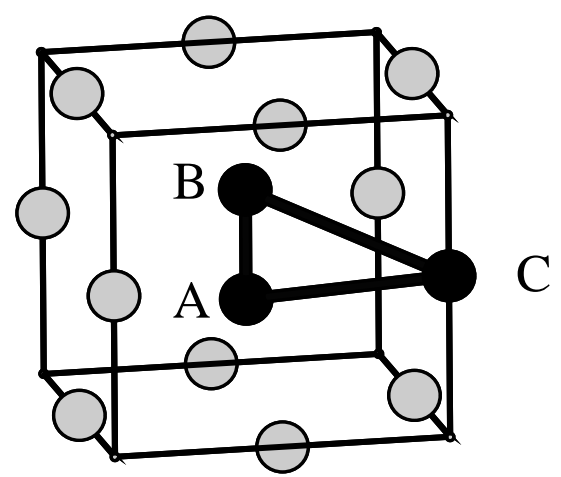

(c)

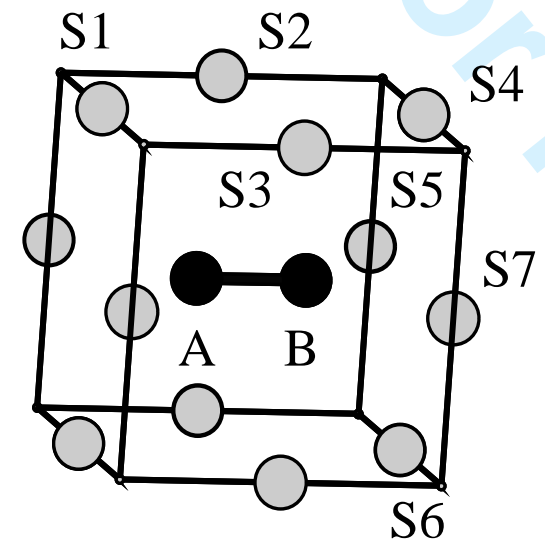

(d)

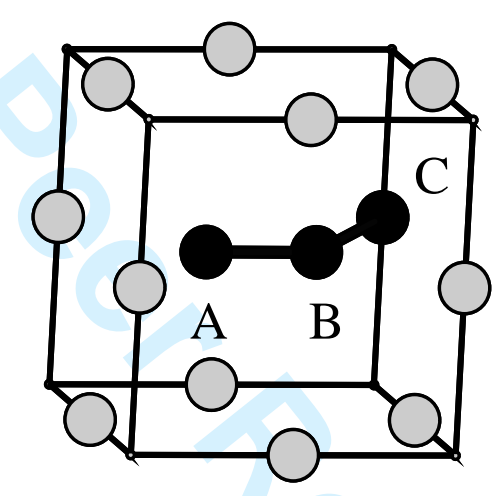

(e)

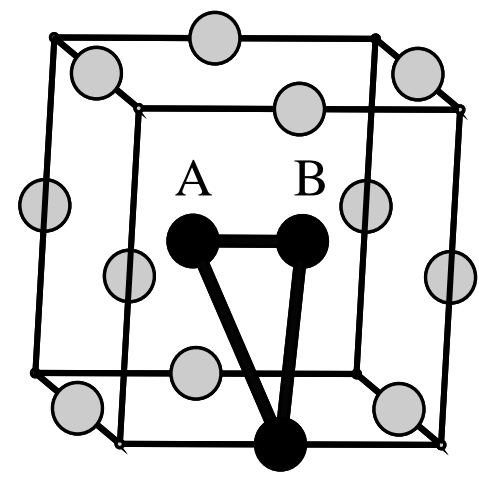

(f) $\mathrm{C}$

positive $\mathrm{X}$ direction

Figure 1: 


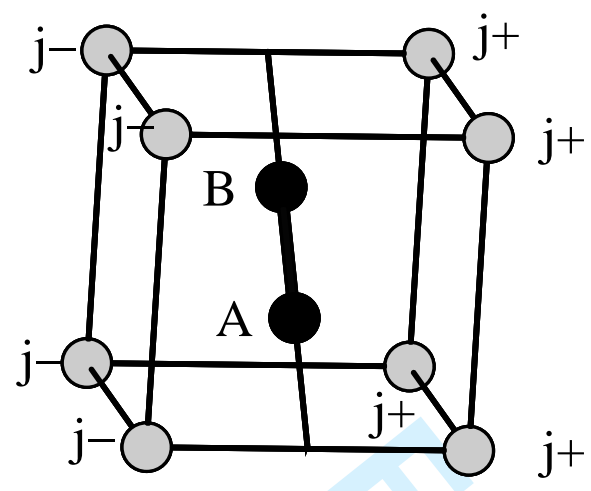

(a)

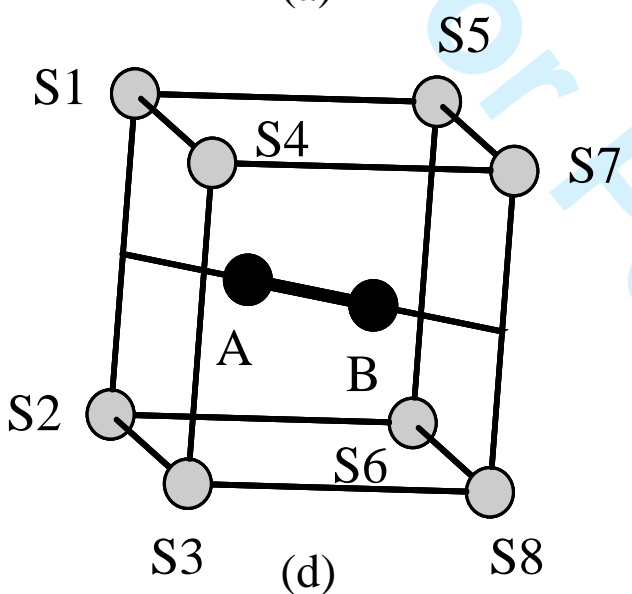

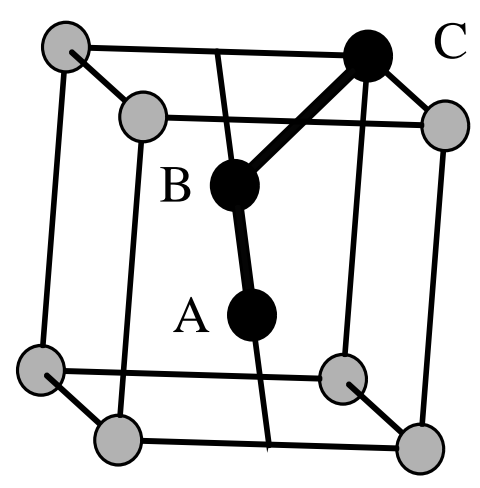

(b)

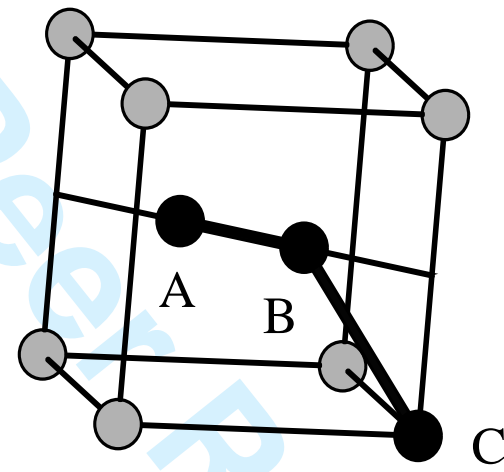

(e)

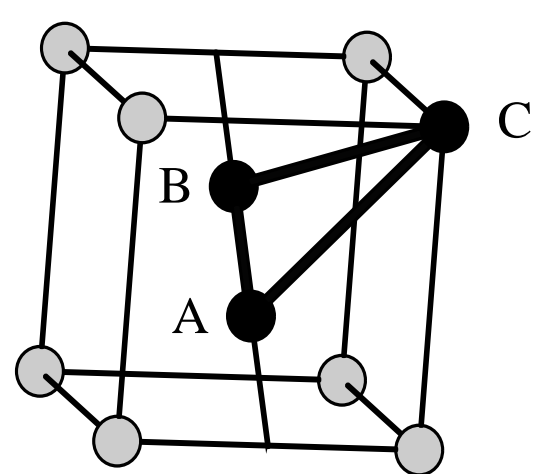

(c)

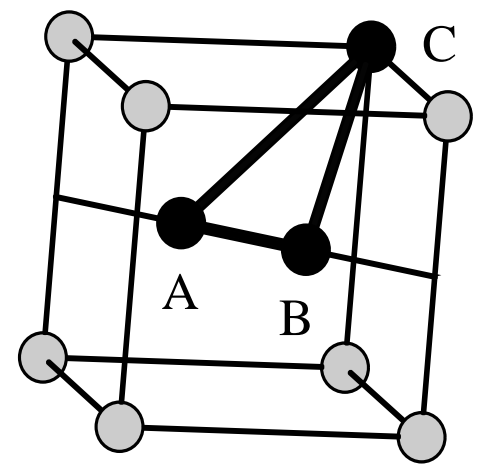

(f)

Figure 2: 


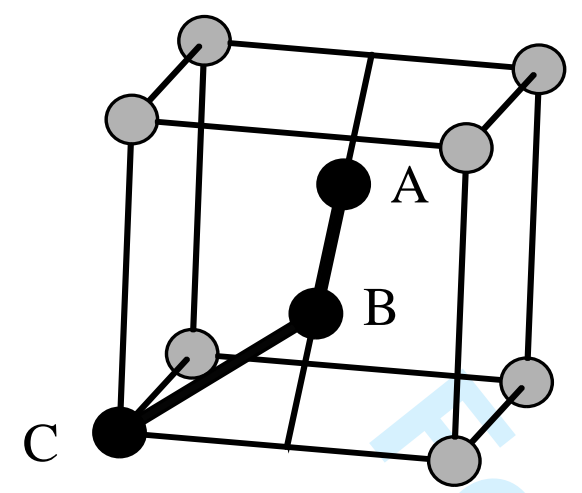

(a)

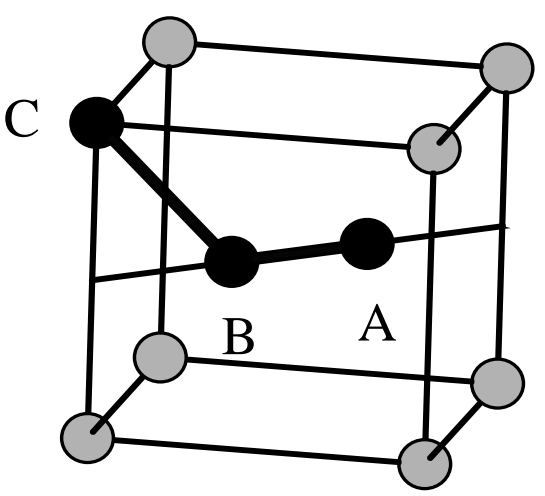

(b)

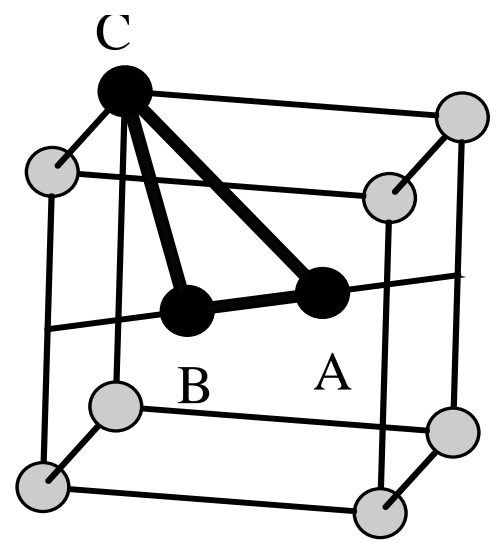

(c)

Figure 3:

http://mc.manuscriptcentral.com/pm-pml 

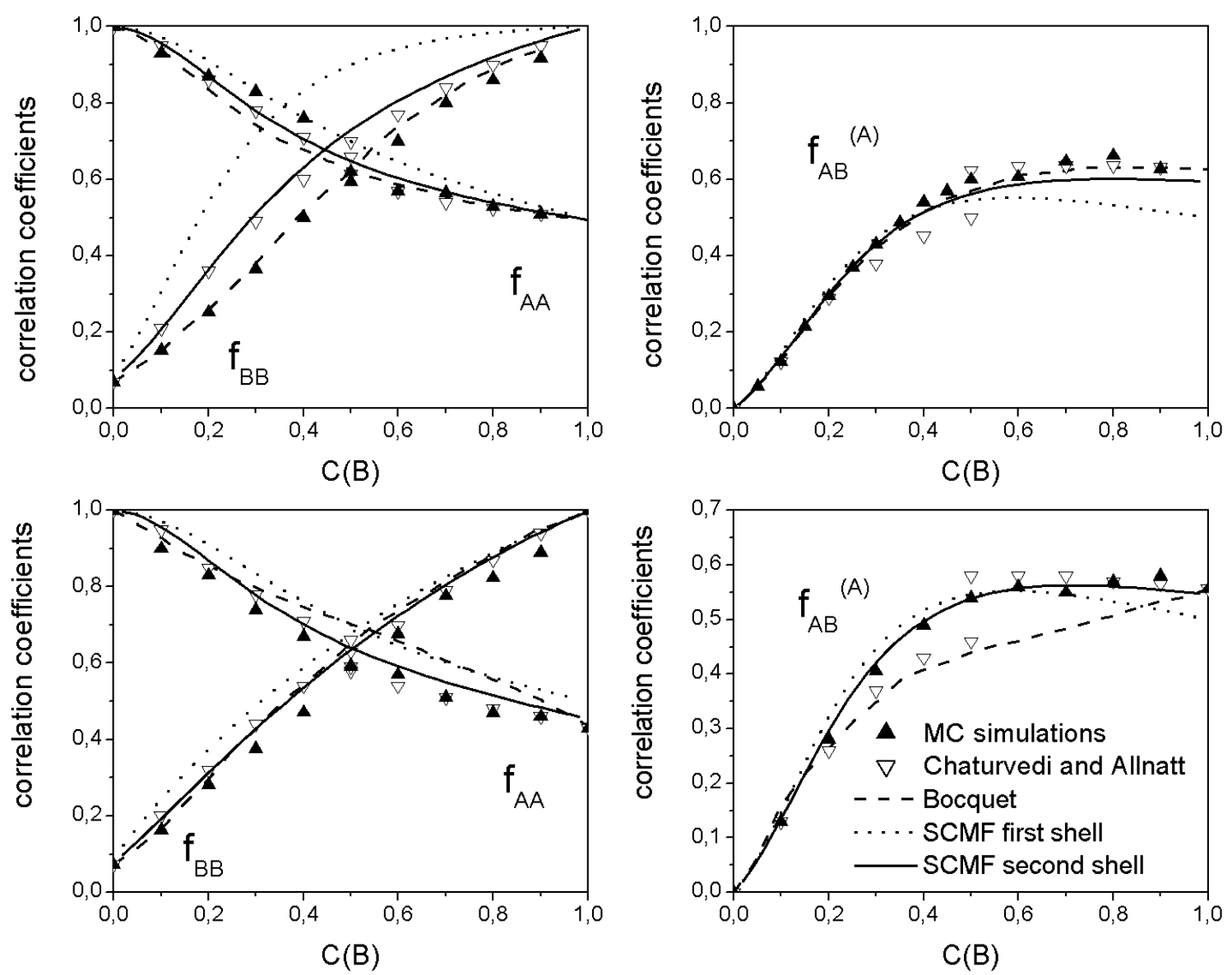

Figure 4:

http://mc.manuscriptcentral.com/pm-pml 

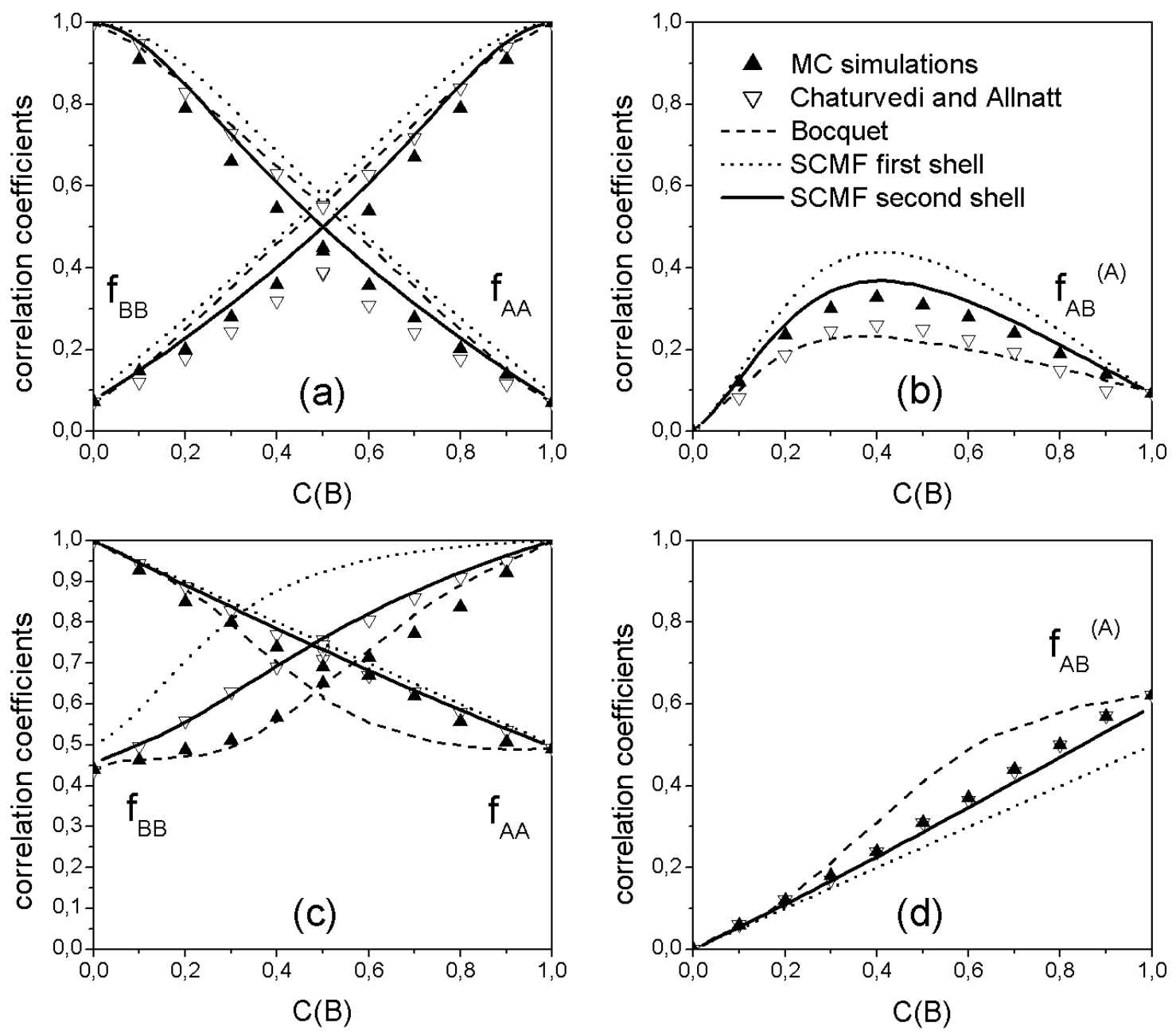

Figure 5:

http://mc.manuscriptcentral.com/pm-pml 

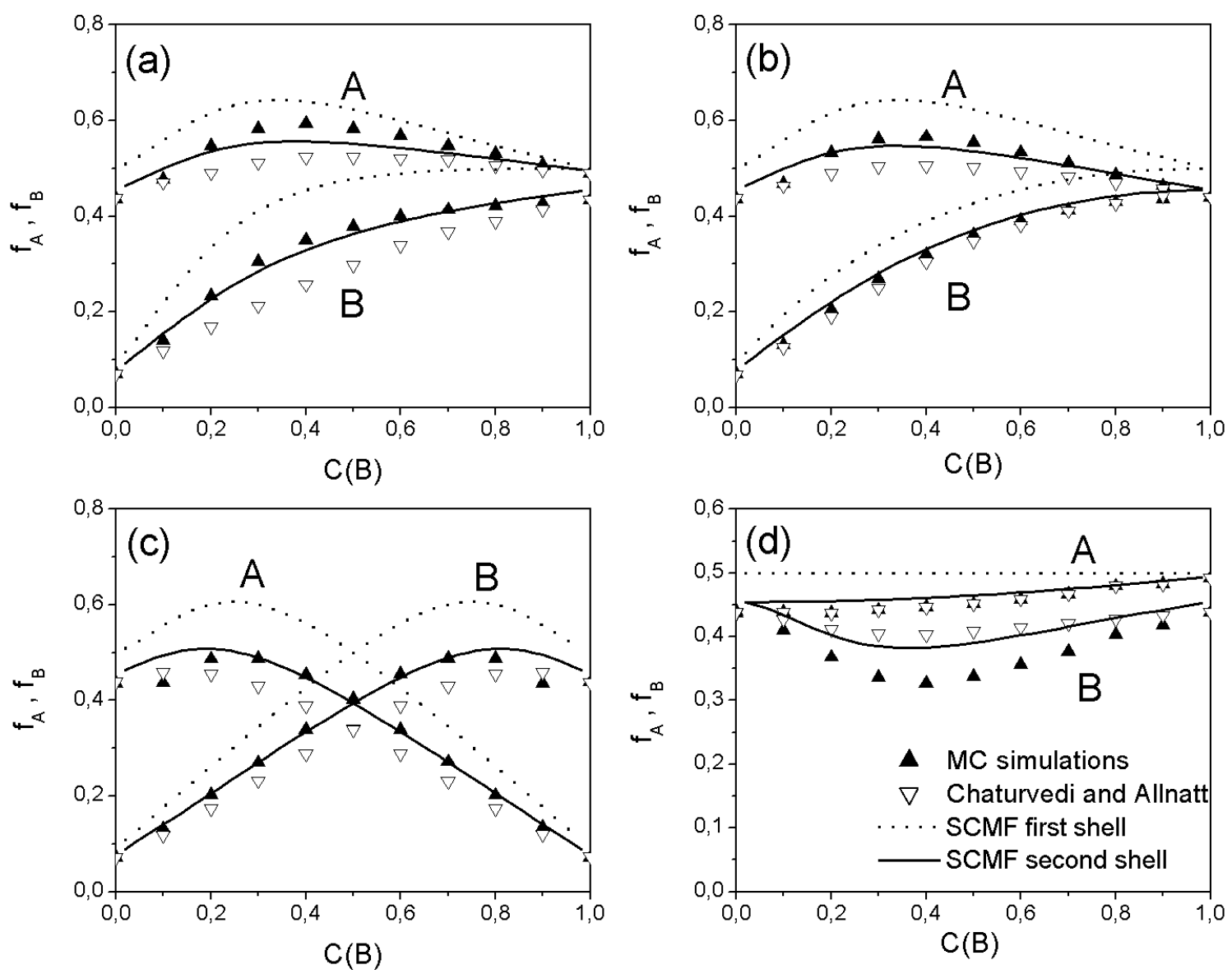

Figure 6:

http://mc.manuscriptcentral.com/pm-pml 

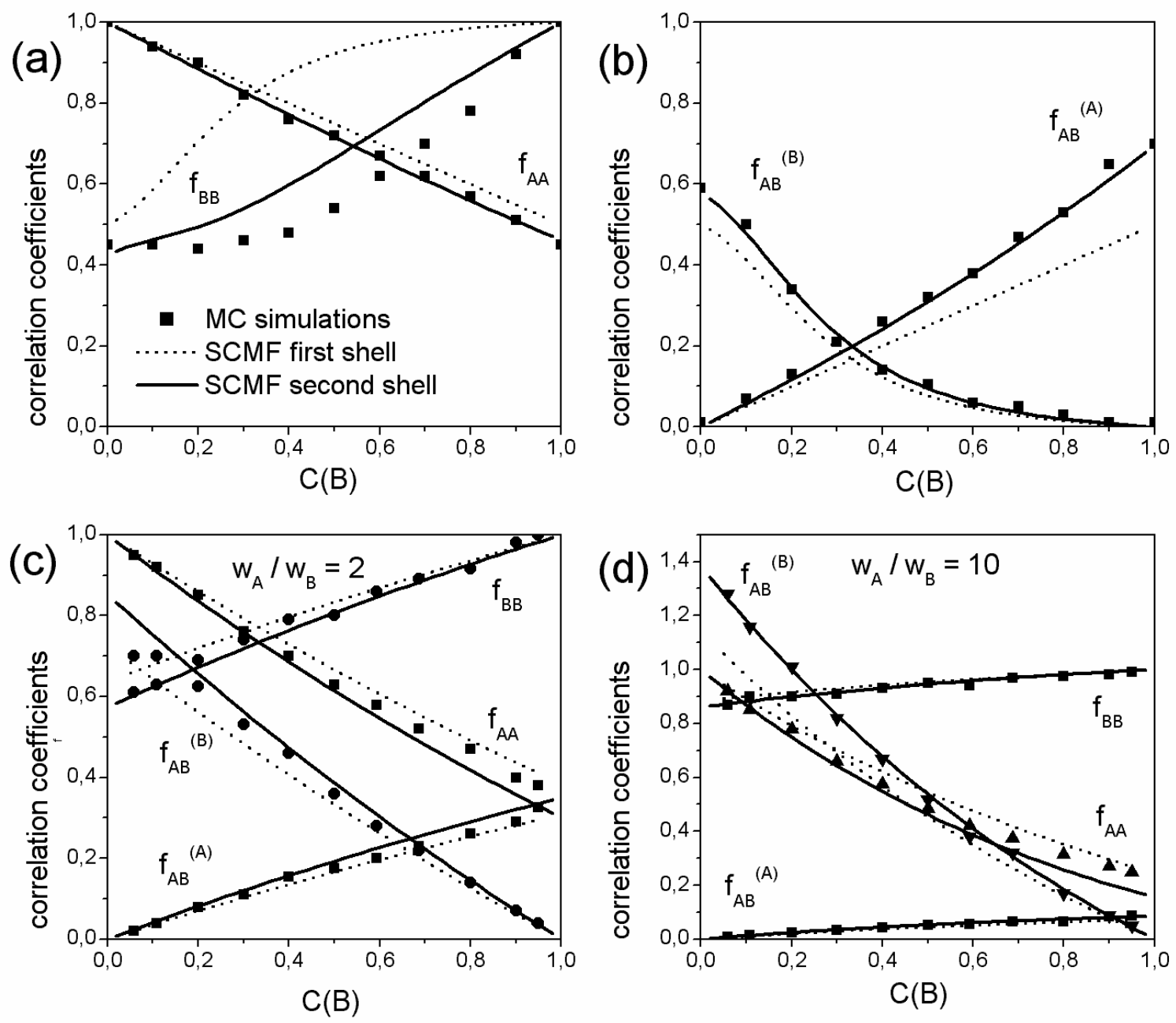

Figure 7: 


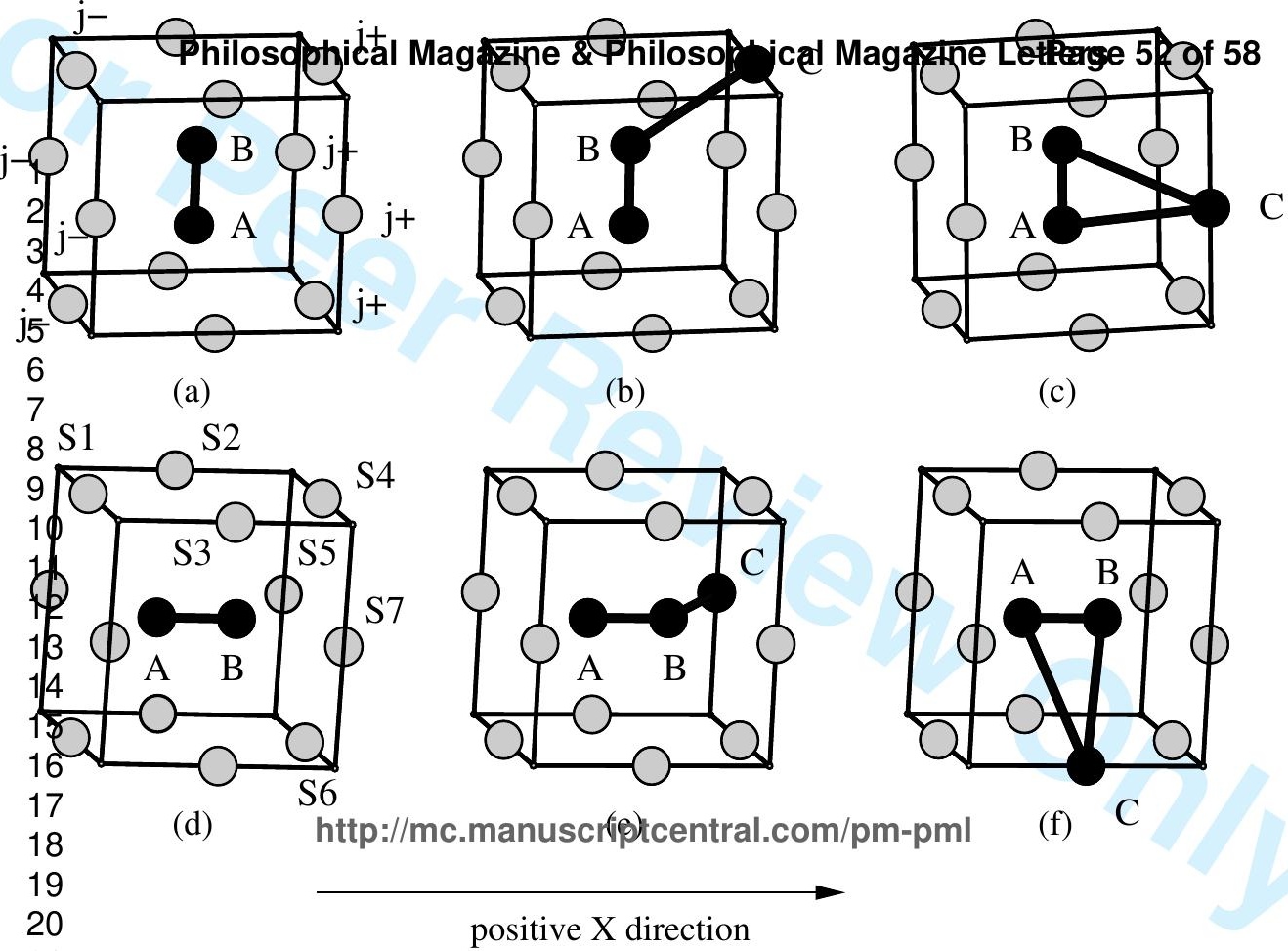




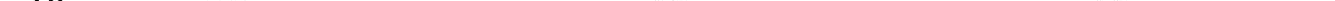




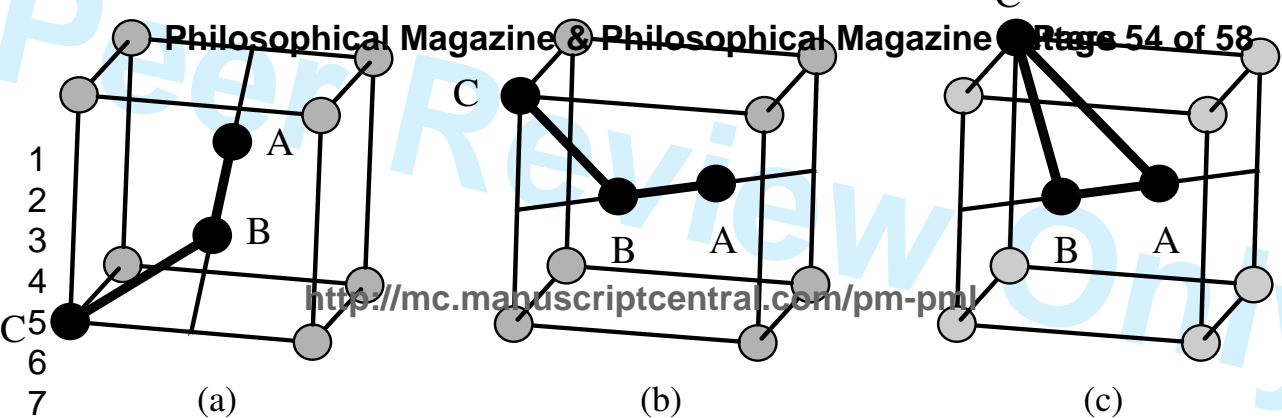



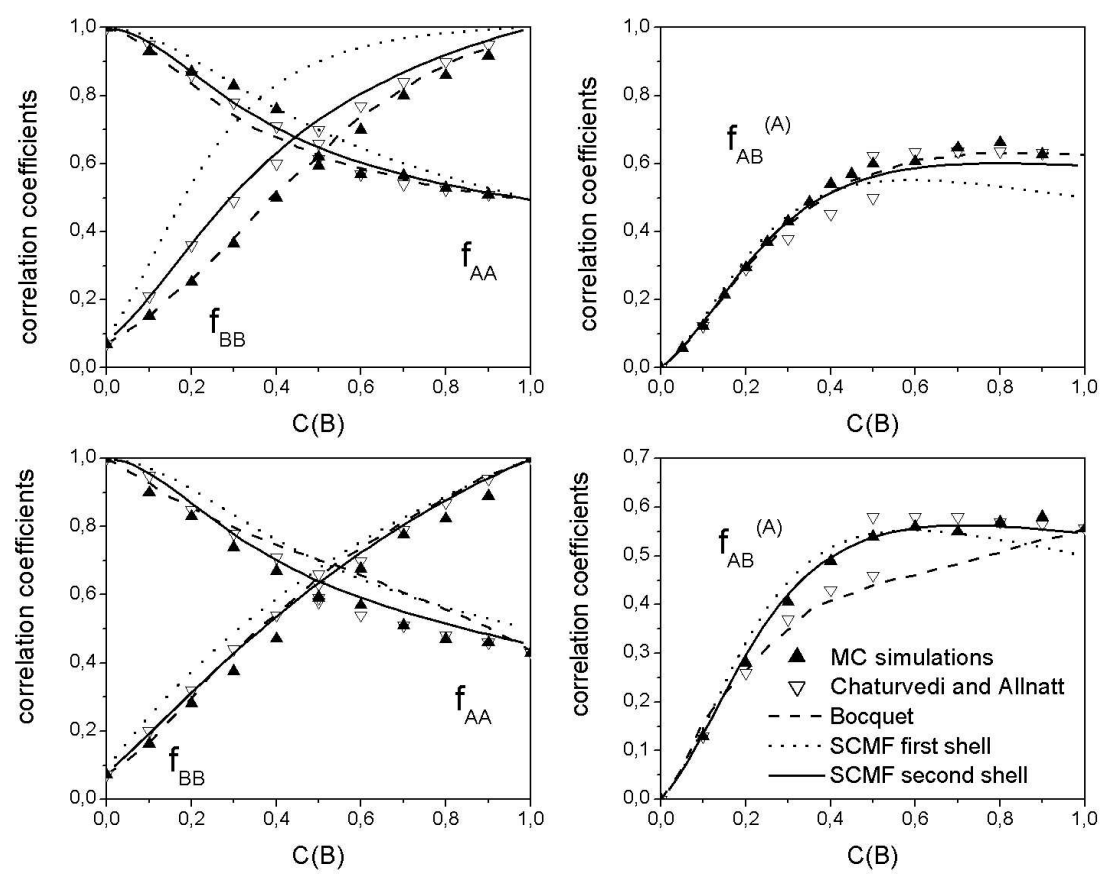

$288 \times 201 \mathrm{~mm}(150 \times 150 \mathrm{DPI})$ 

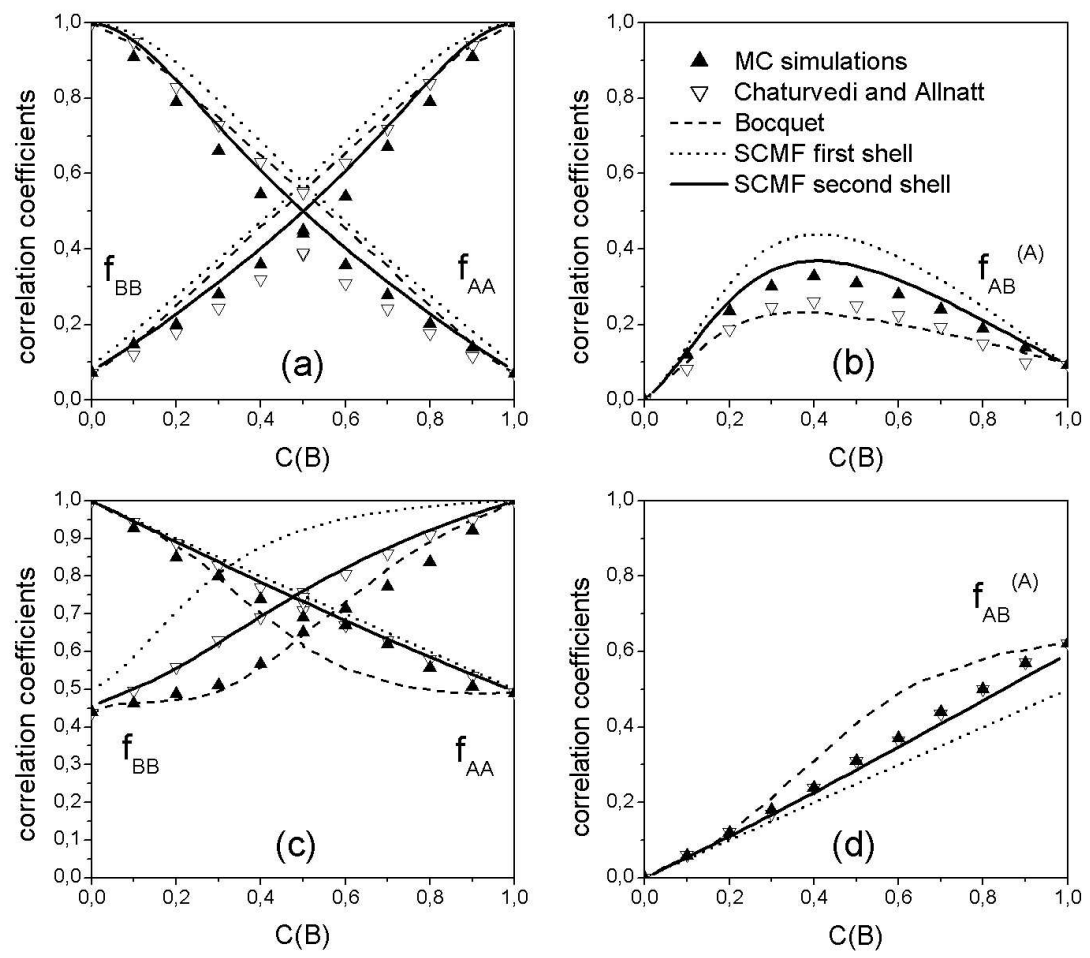

$279 \times 215 \mathrm{~mm}(150 \times 150 \mathrm{DPI})$ 

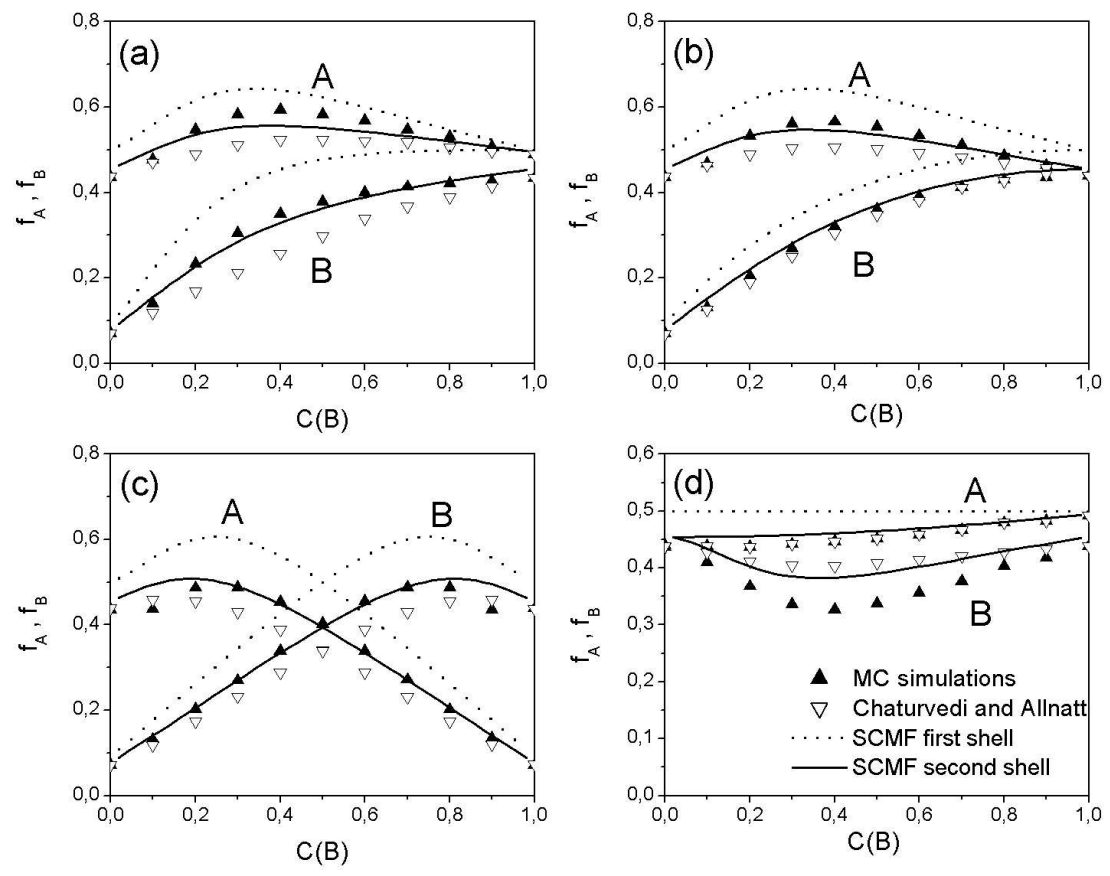

$288 \times 201 \mathrm{~mm}(150 \times 150 \mathrm{DPI})$ 

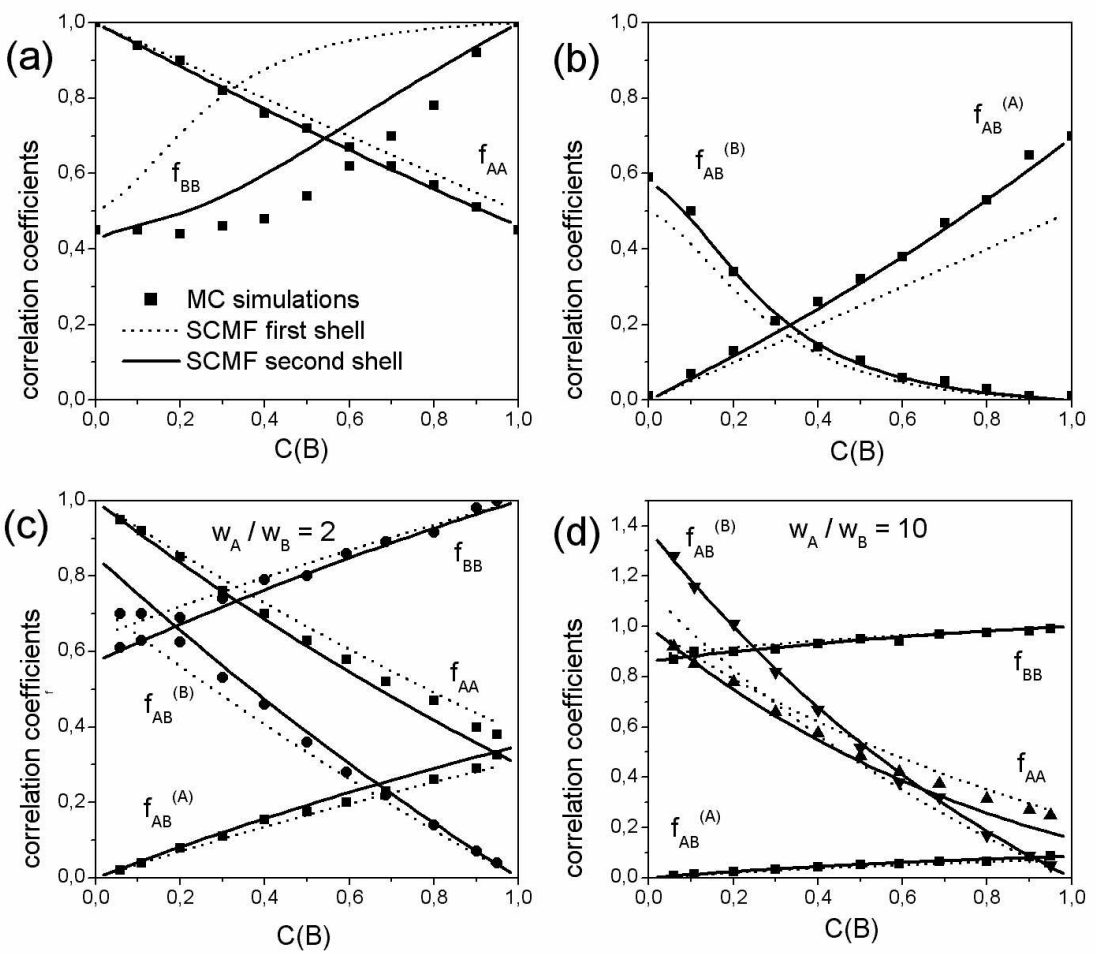

$279 \times 215 \mathrm{~mm}(150 \times 150 \mathrm{DPI})$ 